\title{
Long-range gap junctional signaling controls oncogene-mediated tumorigenesis in Xenopus laevis embryos
}

\author{
Brook T. Chernet ${ }^{1}$, Chris Fields ${ }^{2}$ and Michael Levin ${ }^{1 *}$ \\ ${ }^{1}$ Department of Biology, Tufts Center for Regenerative and Developmental Biology, Tufts University, Medford, MA, USA \\ 2 Independent Researcher, Sonoma, CA, USA
}

\section{Edited by:}

Michal Cifra, Academy of Sciences of the Czech Republic, Czech Republic

\section{Reviewed by:}

Leigh Anne Swayne, University of Victoria, Canada

Richard H. W. Funk, Technische Universität Dresden, Germany Mariano Bizzarri, University La Sapienza Roma, Italy

*Correspondence:

Michael Levin, Department of Biology, Tufts Center for

Regenerative and Developmental Biology, Tufts University, 200 Boston Avenue, Suite 4600 Medford, MA 02155, USA

e-mail: michael.levin@tufts.edu
In addition to the immediate microenvironment, long-range signaling may be an important component of cancer. Molecular-genetic analyses have implicated gap junctions-key mediators of cell-cell communication-in carcinogenesis. We recently showed that the resting voltage potential of distant cell groups is a key determinant of metastatic transformation and tumor induction. Here, we show in the Xenopus laevis model that gap junctional communication (GJC) is a modulator of the long-range bioelectric signaling that regulates tumor formation. Genetic disruption of GJC taking place within tumors, within remote host tissues, or between the host and tumors significantly lowers the incidence of tumors induced by KRAS mutations. The most pronounced suppression of tumor incidence was observed upon GJC disruption taking place farther away from oncogene-expressing cells, revealing a role for GJC in distant cells in the control of tumor growth. In contrast, enhanced GJC communication through the overexpression of wild-type connexin Cx26 increased tumor incidence. Our data confirm a role for GJC in tumorigenesis, and reveal that this effect is non-local. Based on these results and on published data on movement of ions through GJs, we present a quantitative model linking the GJC coupling and bioelectrical state of cells to the ability of oncogenes to initiate tumorigenesis. When integrated with data on endogenous bioelectric signaling during left-right patterning, the model predicts differential tumor incidence outcomes depending on the spatial configurations of gap junction paths relative to tumor location and major anatomical body axes. Testing these predictions, we found that the strongest influence of GJ modulation on tumor suppression by hyperpolarization occurred along the embryonic left-right axis. Together, these data reveal new, long-range aspects of cancer control by the host's physiological parameters.

Keywords: cancer, connexin, ion channel, membrane voltage, bioelectricity, long-range signaling, left-right asymmetry

\section{INTRODUCTION}

Consistent pattern formation during embryogenesis and organ regeneration requires the coordination of cell activities and information across large distances in vivo. Alongside gradients of extracellular secreted molecules, functions an important system of direct cell to cell communication. Gap junctions are intercellular channels that allow small molecule-mediated signals to pass directly from the cytoplasm of one cell to its neighbors (Goodenough et al., 1996; Bruzzone et al., 1996a,b). Connexins, subunits that make up gap junctions (GJs), control the transfer of a wide range of cellular molecules based on their charge, size, and shape (Loewenstein, 1979, 1981; Goldberg et al., 2004), serving as true electrical synapses among non-neural cells (Bennett, 1997; Pereda et al., 2013; Rash et al., 2013). Due to their rich combinatorial properties and gating regulation, gap junction function can implement complex circuits with properties such as memory (Palacios-Prado and Bukauskas, 2009;
Pereda et al., 2013). Many biological processes have evolved to take advantage of these versatile elements, using GJs to regulate differentiation, proliferation, and apoptosis (Krysko et al., 2005; Wong et al., 2008), as well as morphogenetic signaling in many contexts, including development of the brain, limb, bone, tooth, heart, and the left-right axis (Levin, 2007; Wong et al., 2008). Not surprisingly, loss of morphogenetic control due to defective gap junctional communication (GJC) has been implicated in numerous disorders, including hearing impairment (Rabionet et al., 2000), neural tube defects (Ewart et al., 1997), parasitic infection (Vega et al., 2013), cardiovascular diseases (Jongsma and Wilders, 2000), and other genetic syndromes (Zoidl and Dermietzel, 2010). Interestingly, while GJC is most often thought of as mediating local interaction, it can also provide long-range morphogenetic coordination on the scale of the whole organism, for example during the establishment of polarity in regenerating planaria (Oviedo et al., 2010) and 
left-right pattering of Xenopus and chick (Levin and Mercola, 1998, 1999).

A major area in which GJC has been implicated is tumorigenesis (Yamasaki et al., 1995, 1999; Ruch and Trosko, 2001; Trosko, 2005; King and Bertram, 2005; Mesnil et al., 2005). A role for GJs, as mediators of cell-cell signaling and information exchange, is consistent with a view of cancer as a developmental disorder-a derangement of the interaction of cells with the normally tight field of patterning controls of the body (Tsonis, 1987; Pierce and Speers, 1988; Clark, 1995; Dean, 1998; Rubin, 2006; Bissell and Hines, 2011; Marongiu et al., 2012). Alongside cell-autonomous mutations in so-called cancer stem cells, tumorigenesis and progression are controlled by biophysical properties of the surrounding microenvironment (Chernet and Levin, 2013a) and even by neural inputs (Scharrer, 1953; Pawlowski and Weddell, 1967; Magnon et al., 2013). The molecular nature of the processes by which surrounding cells regulate transformation and metastasis is an important area of research today.

By regulating the spread of morphogenetic signals, GJs are an ideal candidate for keeping individual cell activities coordinated toward the anatomical needs of the host, or conversely, allowing confounding signals that may induce tumorigenesis (Levin, 2011, 2012b). Disruption in the function of GJs is implicated in a number of cancers (Yamasaki et al., 1995; Duflot-Dancer et al., 1997; Yamasaki et al., 1999; Ruch and Trosko, 2001; Mesnil et al., 2005; Sirnes et al., 2012). For example, Cx26-deficient mice exhibit a 25 -fold increased incidence of spontaneous liver tumors (Temme et al., 1997). Moreover, tumor incidence is higher, and clinical prognosis is worse, when cells are gap-junctionally isolated by pharmacological agents or genetic mutation (Loewenstein and Kanno, 1966; Loewenstein, 1979; Rose et al., 1993; Mesnil et al., 2005).

Most clinically-relevant tumor cells are known to exhibit down-regulation in connexin expression, leading to the disruption of effective cell:cell communication (Soroceanu et al., 2001; Gee et al., 2003; Mesnil et al., 2005; Talbot et al., 2013). However, in some some studies, enhanced GJC was suggested as a tumor promoting factor (Saito-Katsuragi et al., 2007; Naoi et al., 2007; Elzarrad et al., 2008; Haass et al., 2010). Breast cancer and melanoma cells take advantage of these connexins to enhance their metastatic potential in Stoletov et al. (2013). Together, the data suggest that it is imperative to understand the signaling mediated by GJs and the information passed among normal cells that could promote (Rose and Wallingford, 1948; Lewalle et al., 1998; Zhang et al., 2003; Donahue et al., 2003), or normalize (Hendrix et al., 2007), cancer in vivo. How might GJC regulate tumorigenesis in the context of tissue and organ patterning?

One carrier of the morphogenetic cues that go awry during tumorigenesis may be current- the movement of charged ions. Gradients of resting potentials established by ion channels and pumps in the cell membrane are now known to be instructive patterning cues that regulate cell behavior during pattern formation (Levin, 2012a; Tseng and Levin, 2013; Levin, 2013, 2014), and gap junctions sculpt the distribution of iso-potential cell fields by allowing specific cells to equalize their trans-membrane voltages $\left(\mathrm{V}_{\mathrm{mem}}\right)$ in response to various physiological signals. Bioelectric gradients have already been implicated in the control of metastasis (Morokuma et al., 2008; Blackiston et al., 2011) and oncogene-mediated tumorigenesis (Lobikin et al., 2012; Chernet and Levin, 2013a,b, 2014), while specific ion channels are becoming increasingly recognized as oncogenes and important drug targets (Diss et al., 2005; Fraser et al., 2005; Arcangeli et al., 2009; House et al., 2010; Yildirim et al., 2012; Arcangeli et al., 2012; Yang and Brackenbury, 2013; Than et al., 2014; Pardo and Stuhmer, 2014). Interestingly, the resting potentials of distant cells are critical for oncogene-dependent tumorigenesis: modulation of ion channels in locations quite distant to oncogene expressing cells in Xenopus tadpoles significantly reduces the incidence of tumors. This effect is mediated by a butyrate-based mechanism that regulates oncogene-mediated tumorigenesis via histone deacetylase activity (Chernet and Levin, 2013a,b, 2014), but the spatial dynamics of butyrate signaling in this context remain to be elucidated. The known role for ion flows and resting potentials in cancer suggests the possibility that gap junctions participate in bioelectric regulation during carcinogenesis and/or neoplastic progression.

To investigate the complex interplay of physiological and genetic signals in tumorigenesis, we pursued a combination of modeling and experiment to probe the spatial relationships between tumorigenesis, voltage properties, and GJ paths in vivo. We recently showed that ion channel-mediated changes in $\mathrm{V}_{\text {mem }}$ can modulate the tumorigenicity of human oncogenes misexpressed in the Xenopus laevis embryo (Chernet and Levin, 2013b, 2014). Here, we use this assay to investigate the interplay between bioelectric controls and gap-junctional connectivity in vivo. Unexpectedly, we found that disruption of cell:cell communication via $\mathrm{H} 7$ - a chimeric connexin construct that is known to inhibit GJC in Xenopus (Paul et al., 1995; Levin and Mercola, 1998) - is able to suppress tumor formation. Remarkably, the suppression effect was observed regardless of where GJC disruption was occurring (host-wide, within tumors only, or away from tumors). Indeed, the most pronounced suppression was recorded for GJC disruption taking place nonlocally to oncogene-expressing cells, revealing a role for distant cell:cell communication in tumorigenesis. Conversely, enhanced GJC within tumors or their microenvironment, achieved via the mis-expression of a constitutively permeable junction-forming connexin Cx26 (Levin and Mercola, 1998), increased tumor incidence. Together, these data suggest that GJC is a mediator of both local (within tumors) and long-range (within the microenvironment and the host) signaling and that specific patterns of physiological isolation may be necessary for tumor suppression. Here we also formulate a mechanistic, quantitative model consistent with these data and with prior work on endogenous left-right asymmetric voltage gradients, and test key predictions of this model. Based on these data, we suggest that exploiting the bioelectrical signaling that occurs through electrical synapses among somatic cells represents an important target for cancer therapy that is distinct from the targeting of individual channels.

\section{MATERIALS AND METHODS ANIMAL HUSBANDRY}

$X$. laevis eggs were fertilized in vitro, and embryos were cultured according to standard protocols (Sive et al., 2000), in 0.1X Modified Marc's Ringers (MMR; pH 7.8) with $0.1 \%$ Gentamicin. Xenopus embryos were housed at $14-18^{\circ} \mathrm{C}$ and staged according 
to Nieuwkoop and Faber (1967). All experimental procedures involving the use of animals for experimental purposes were approved by the Institutional Animal Care and Use Committees (IACUC) and Tufts University Department of Lab Animal Medicine (DLAM) under the protocol number M2014-79.

\section{MICROINJECTION}

Fertilized Xenopus embryos were transferred into mesh-bottomed dishes with 3\% Ficoll and injected with capped, synthetic mRNAs (made using the Ambion Message Machine kit) dissolved in water at the stages indicated. The doses per cell were KRAS ${ }^{G 12 D}$ (Le et al., 2007), 40 pg; H7 (Paul et al., 1995), 70 pg; Cx26 (Levin and Mercola, 1998), $500 \mathrm{pg}$; and $\beta$-gal (lineage tracer), $400 \mathrm{pg}$. Two hours after injection, embryos were transferred into $0.75 \mathrm{X} \mathrm{MMR}$ for $45 \mathrm{~min}$ before they were washed and cultured in 0.1X MMR until desired stage was reached. KRAS $S^{G 12 D}$-injected embryos were raised to stage $\sim 35$, and scored for the presence of tumors using bright field microscopy as described in Chernet and Levin (2013b).

\section{TESTING GJC}

GJC was assessed between tumors and the host using a 1:1 mixture of Rhodamine-Lysinated Dextran (RLD, $10 \mathrm{kDa}$, Life Technologies) and Lucifer Yellow (LY, $0.522 \mathrm{kDa}$, Life Technologies) (Figure 1). 16 cell embryos injected with $K R A S^{G 12 D}$, RLD, and LY were allowed to grow to stage $\sim 35$. Cells exhibiting LY signal (which passes through GJs) in the absence of RLD (which does not pass through GJs) signal reveal an open gap-junctional communication (cells with both LY and RLD signal indicate regions that have acquired the two molecules through cell division and/or migration). RLD and LY were detected in live embryos using TRITC and Lucifer Yellow filtersets, respectively, on an Olympus BX61 spinning-disk confocal microscope with Hamamatsu ORCA digital CCD camera.

\section{STATISTICAL ANALYSIS}

Data were expressed as the mean unless otherwise noted. The differences between treatment groups were analyzed using Student's $t$-test and $X^{2}$ test, and the null hypothesis was rejected at the 0.05 level.

\section{PREDICTIVE MODELING}

A quantitative model of embryonic growth and response to microinjection treatments was developed in two stages that model different processes (Figures 4, 5). The first-stage "leftright synchronization" model was implemented using JavaScript and the HTML5 "canvas" function. Details of this model are described below (Section A two-stage quantitative model describes the dynamics between $V_{\mathrm{mem}}$, GJC, and tumor formation); the model is interactive, and can be manipulated and the source code can be examined at http://chrisfieldsresearch.com/convergencedemo.htm (also seen in Supplement 1). The second-stage "leftright communication" model comprises the implications of a set of assumptions that specify a control network as described below (Section A two-stage quantitative model accurately predicts the dynamics between $V_{\text {mem }}$, GJC, and tumor formation). Numerical predictions were calculated manually from these assumptions.

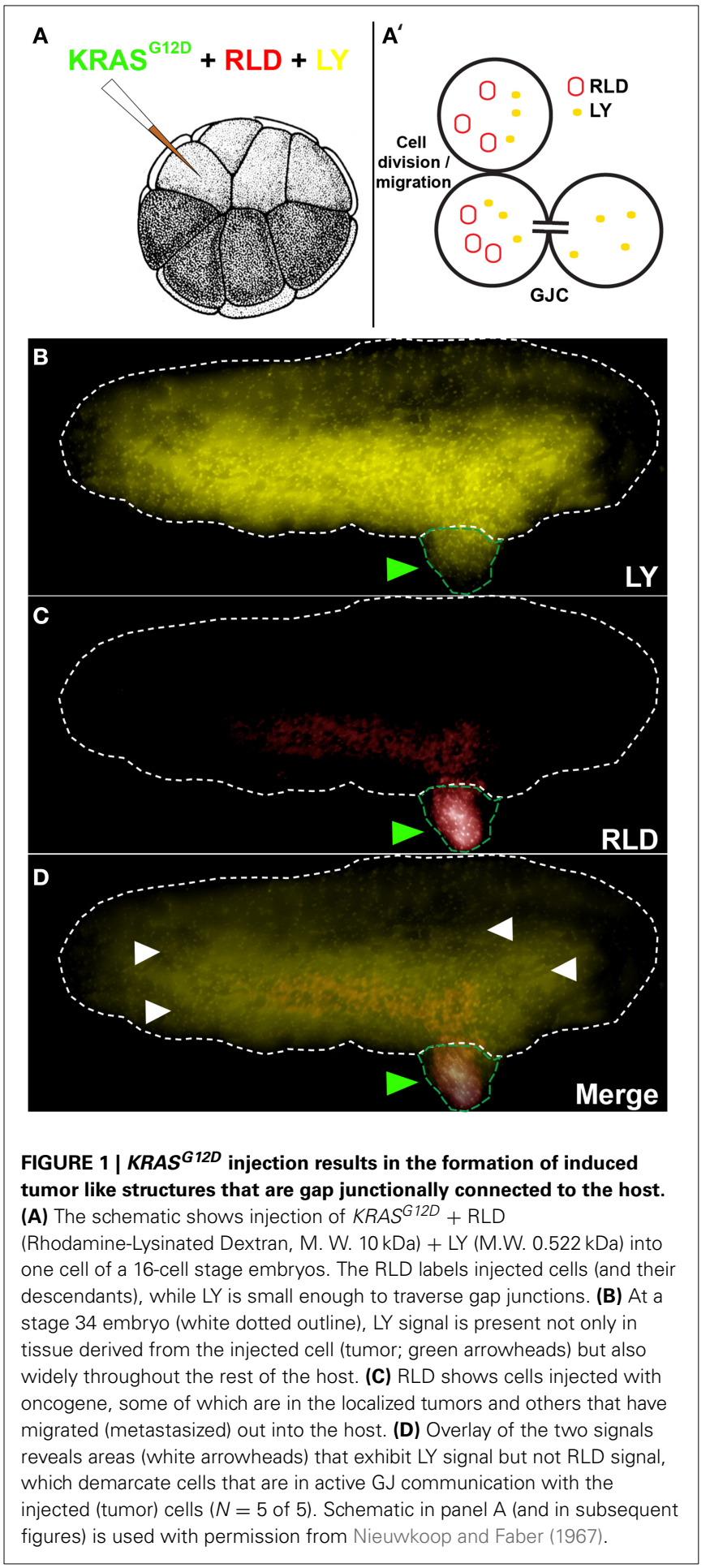

\section{RESULTS}

\section{KRAS $^{G 12 D}$-INDUCED TUMORS ARE JUNCTIONALLY COUPLED TO} NORMAL HOST CELLS

To study the role of GJC in oncogene-mediated tumorigenesis, we made use of a simple assay that utilizes expression of an exogenous human oncogene in X. laevis embryos (Figure 1A). This model system is ideal for probing connexin-based GJC 
(Swenson et al., 1989; Barrio et al., 1997; Cao et al., 1998; Lee et al., 2009) and has been used to study GJC-mediated morphogenesis and large-scale patterning (Warner, 1992; Levin and Mercola, 1998; Levin, 2002). The oncogene KRAS ${ }^{G 12 D}$ (Le et al., 2007) was used to induce Xenopus tumors (Figures 1B-D, green arrowhead). These exhibit the same major hallmarks of tumors as do their mammalian counterparts: increased mitotic activity, induced vasculogenesis, disorganization of normal cellular architecture, increased hypoxia, acidic microenvironment, and ability to illicit innate immune response (Chernet and Levin, 2013b, 2014). We began by testing GJC between induced tumors and the host, which revealed considerable spreading of the small GJC-permeant tracer Lucifer Yellow (Figures 1B,D; yellow signals, white arrowheads) away from descendants of one of the 16 progeny cells that were injected with oncogene mRNA and a large, GJC-impermeant Rhodamine Dextran (5 out of 5 tested; Figures 1C,D; red signal). Having observed active GJC between the tumor and surrounding host tissue, we began functional studies by investigating how the growth of these oncogene-induced tumors is affected by spatially targeted disruption of GJC. All of the reagents used below were titered to levels low enough that the normal background processes of embryogenesis and growth proceeded normally.

\section{DISRUPTION OF GJC REDUCES TUMOR INCIDENCE}

To determine the effect of GJC disruption on tumorigenesis in vivo, mRNAs of dominant-negative connexin $\mathrm{H} 7$ (Paul et al.,
1995; Levin and Mercola, 1998) and KRAS ${ }^{G 12 D}$ were injected in $X$. laevis embryos. The oncogene and GJC disrupting constructs were introduced in different spatial arrangements to investigate what happens when GJC is disrupted host-wide, within tumors, and away from tumors. Taking advantage of the embryonic fatemap (Dale and Slack, 1987; Moody and Kline, 1990), we were able to control which cells received oncogene mRNA and which cells were inhibited with respect to GJC. To achieve suppression of GJC within the tumor ("local" mode), H7 mRNA was injected into 1 cell of a 2-cell embryo and the KRAS ${ }^{G 12 D}$ mRNA was introduced into one of the progeny of that cell (at the 16-cell stage). For remote ("long range" mode) GJC suppression in tissue outside the tumor, H7 mRNA was injected into 1 cell of a 2-cell embryo, which was raised to the 16-cell stage and then injected with KRAS ${ }^{G 12 D}$ mRNA into one of the cells on the opposite side of the embryo from the cells bearing the H7. For host-wide GJC disruption, both of the 2 cells of the 2-cell embryo were injected with $\mathrm{H} 7$, and $K R A S^{G 12 D}$ mRNA was then injected in 1 of the 16 progeny cells. Figure 2 shows the various combinations of which sides of the embryo received the GJ blocker (H7) and which received the oncogene (KRAS ${ }^{G 12 D}$ ).

Compared to tumor incidences observed in the baseline $K R A S^{G 12 D}$-only injected embryos (Figure 2; treatment mode A), local (treatment mode B) and host-wide (treatment mode D) disruption of GJC showed significant suppression of tumor formation by 6.6 and $5.8 \%$, respectively ( $t$-test; $p<0.05$ ). Interestingly, long-range disruption of GJC (treatment mode C) had the

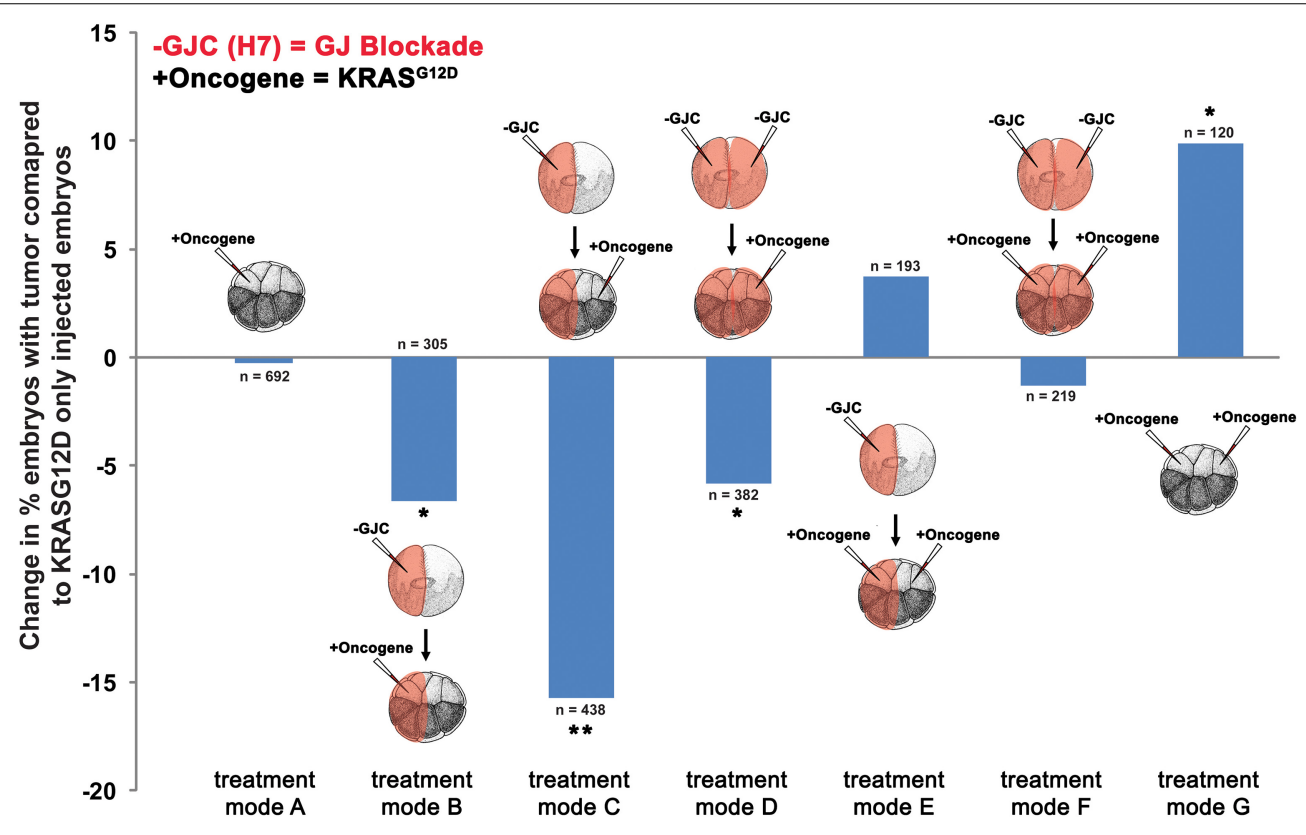

FIGURE 2 | Selective disruption of gap junctional communication (GJC) reduced tumor incidence. To determine the effect of GJC disruption on tumorigenesis, $\mathrm{H} 7$ and $K R A S^{G 12 D}$ injections-aimed at disrupting GJC host-wide, within tumors, and away from tumors-were performed. Compared to KRAS ${ }^{12 D}$-only injected embryos (treatment mode A), treatment modes $B$ and $D$ showed significant decrease in \% embryos with tumor by 6.6 and $5.8 \%$, respectively, implying that $\mathrm{H} 7$ contralateral to the oncogene has no effect if there is $\mathrm{H} 7$ ipselateral to the oncogene. Contralateral $\mathrm{H} 7$ only (treatment mode $\mathrm{C}$ ) significantly reduces the \% embryos with tumor by $15.8 \%$. Doubling the level of oncogene injected along with host-wide introduction of $\mathrm{H} 7$ (treatment mode $\mathrm{F}$ ) does not affect tumor incidence when compared to oncogene-only (one side) injected embryos (treatment mode A), but is able to counter the $9.9 \%$ increase in tumor incidence resulting from excess oncogene introduced (treatment mode G) down to the tumor incidence level of one-side oncogene injection. Similarly, tumor incidence from the excess oncogene introduction can be reduced by $\mathrm{H} 7$ introduction to either side of the embryo (treatment mode E). ${ }^{*} P<0.05,{ }^{* *} P<0.001$; $t$-test. 
strongest suppressive effect, showing a $15.8 \%$ decrease in the number of embryos with tumor ( $t$-test; $p<0.001)$. Injection of H7 host-wide with KRAS ${ }^{G 12 D}$ injected in 2 cells on opposite sides of the 16-cell embryo (Figure 2; treatment mode F) did not affect tumor incidence when compared to KRAS G12D only injected embryos (Figure 2; treatment mode A). However, the host-wide $\mathrm{H} 7$ introduction (Figure 2; treatment mode $\mathrm{F}$ ) reduced tumor incidence resulting from oncogene expression on both sides of the embryo (compare to Figure 2; treatment mode $\mathrm{G})$ by more than $11 \%(t$-test; $p<0.05)$. Similarly, tumor incidence from doubling the oncogene level can be reduced by the introduction of $\mathrm{H} 7$ to only one side of the embryo (treatment mode E). Together, the results imply that GJC disruption suppresses KRAS ${ }^{G 12 D}$-induced tumors: the most pronounced effect on tumor incidence is observed when $\mathrm{H} 7$ is introduced at a distance from the oncogene-bearing cells, unless $\mathrm{H} 7$ is also expressed within the oncogene-expressing cells, in which case suppression is not as effective.

\section{FACILITATED GJC PROMOTES TUMOR FORMATION}

To further test the hypothesis that GJC plays a role in oncogenemediated tumorigenesis, we scored tumor incidence in embryos injected with $\mathrm{Cx} 26$, a short connexin protein which lacks most of the intracellular regulatory region, and thus facilitates GJC by forming constitutively permeable gap junctions (Mesnil et al., 1997; Levin and Mercola, 1998). Similar to the H7 (GJ blockade) experiment, mRNA injections of $\mathrm{Cx} 26$ and $K R A S^{G 12 D}$ aimed at enhancing GJC host-wide, within tumors, and away from tumors were performed (Figure 3). Compared to KRAS ${ }^{G 12 D_{-}}$ only injected embryos (Figure 3, treatment mode A), longrange and host-wide $\mathrm{Cx} 26$ treatments (treatment modes $\mathrm{C}$, $\mathrm{D}, \mathrm{F})$ both showed an increase in number of embryos with tumor by 6.4 to $11.4 \%$. This is in contrast to the $\mathrm{H} 7$ data where modulation of long-range GJC had the most impact on tumor incidence. Interestingly, enhanced GJC within tumors (treatment modes B and E) slightly (but not significantly) decreased tumor incidences when compared to treatment modes A and F, respectively. Together, these data indicate that facilitating GJC host-wide and in the tumor microenvironment enhances the tumorigenesis process. In contrast, enhanced GJC within oncogene-expressing cells had minimal effect on tumor formation.

\section{A TWO-STAGE QUANTITATIVE MODEL DESCRIBES THE DYNAMICS BETWEEN $\mathbf{V}_{\text {mem, }}$ GJC, AND TUMOR FORMATION}

The tumor-incidence results were challenging to explain for the following reason. While perturbing GJC in the same side as $K R A S^{G 12 D}$ expression (Figure 2, treatment mode B) reduced

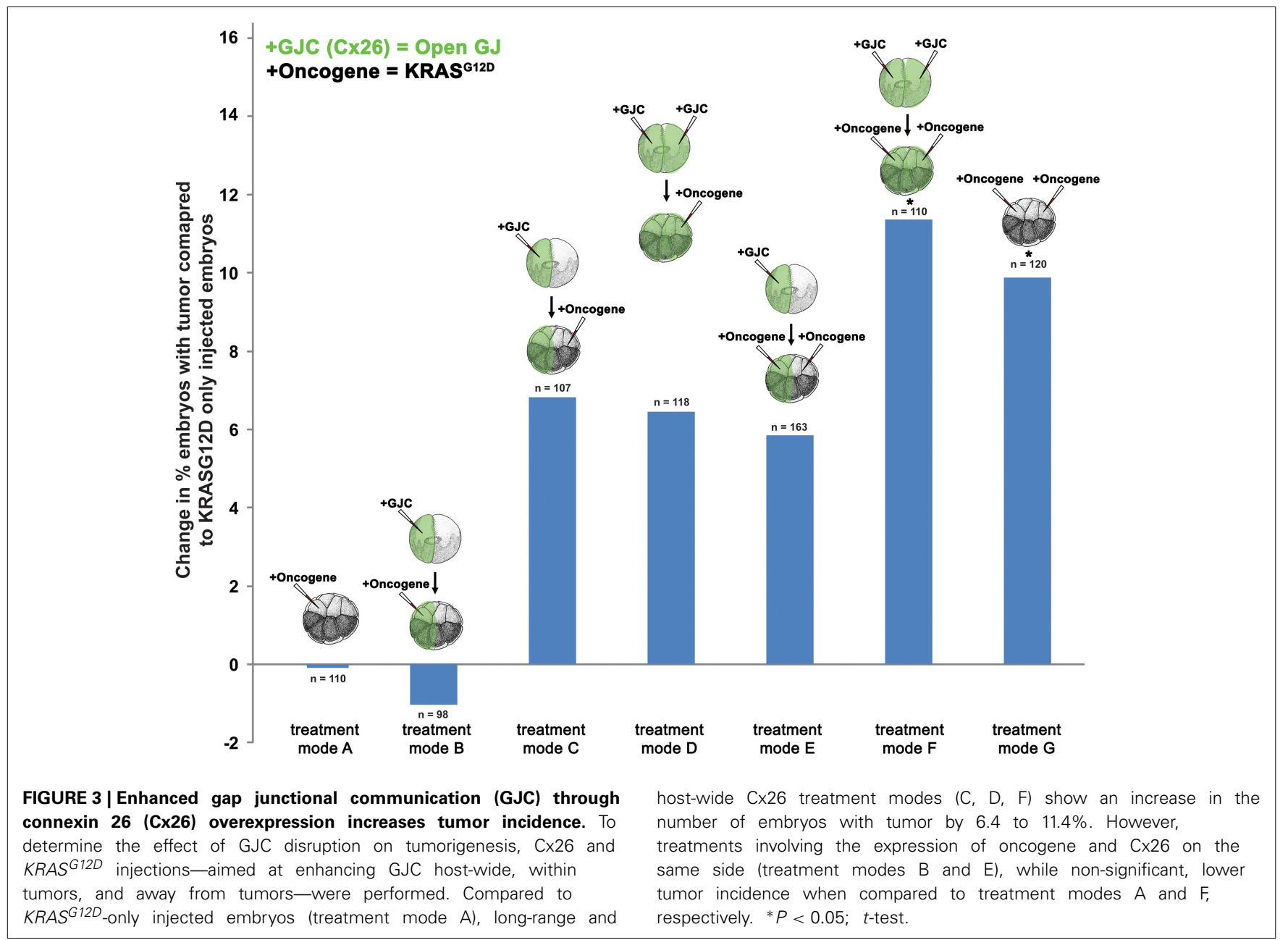


tumor incidence, the effect became more pronounced when the GJC perturbation is on the opposite side of KRAS ${ }^{G 12 D}$ injection (treatment mode $\mathrm{C}$ ); it is puzzling why having $\mathrm{H} 7$ on the opposite side from KRAS ${ }^{G 12 D}$ injection could make the KRAS ${ }^{G 12 D}$ expressing side much less likely to form a tumor. More strikingly, why would perturbing GJC on both sides of the embryo, while only introducing KRAS ${ }^{G 12 D}$ on one side (Figure 2, treatment mode D), have the same effect as perturbing GJC on the KRAS ${ }^{G 12 D}$ injected side only?

In order to mechanistically integrate the main components of our experiments $\left(\mathrm{V}_{\text {mem }}\right.$ manipulated by ion channel injections, GJC manipulated by $\mathrm{Cx}$ injections, and long-range effects on tumors induced by oncogene injections), a two-stage model was developed to explain the tumor-incidence data shown in Figures 2, 3 and to make additional testable predictions. The first stage of this model (Figure 4) establishes regions of different $\mathrm{V}_{\text {mem }}$ within the embryo and provides the input required by the second stage (Figure 5), which shows the dynamics that regulate tumor outcome. The dynamics postulated by the second-stage model require that the left and right sides of the embryo exchange a signal; it is assumed that this alternating signal is implemented, or at least enabled, by coupled oscillations of $\mathrm{V}_{\text {mem }}$ on the two sides as described in more detail below.

Our two-stage model is based on the observation that largescale bioelectric cues can provide patterning information during development (Nuccitelli, 2003a,b; McCaig et al., 2005, 2009; Levin, 2012a; Zhao et al., 2012). Let us call the left and right sides of the Xenopus embryo A and B, in no particular order. Assume that the two sides exchange a long-range signal that controls proper patterning and subsequent maintenance of order, and that both A and B also respond to cell-autonomous and local signals. We hypothesized that it is an interaction between long-range and short-range signals that produces the non-local effects that we see.

If the left and right sides of the embryo are to produce a coherent long-range bioelectric signal, their long-range signaling activity must be coordinated or synchronized. Cells are known, for example, to alternate between hyperpolarization and depolarization with cell cycle (Bregestovski et al., 1992; Arcangeli et al., 1995; Blackiston et al., 2009). If this alternation between hyperpolarization and depolarization was synchronized so that the left side was fully polarized when the right side was fully depolarized and vice-versa, it could produce a coherent long-range signal. The first, "left-right synchronization" stage of our model generates synchronized left and right cell populations from a random initial state. The model assumes a total starting population of 100 cells, corresponding roughly to embryonic cell division seven $\left(2^{7}=128\right)$. Below this number of cells, nearest-neighbor interactions are insufficient to produce global order because too many neighborhoods overlap. The model also assumes an initial left-right bias in the polarization state of the cells [as has been observed experimentally to derive from consistently-asymmetric localization of ion channels on the left and right sides of the 2-cell embryo as it divides (Levin et al., 2002; Qiu et al., 2005; Adams et al., 2006; Morokuma et al., 2008)]. The left-right bias was treated as a free parameter and varied to optimize model behavior. The cells are assumed to occupy a planar array representing

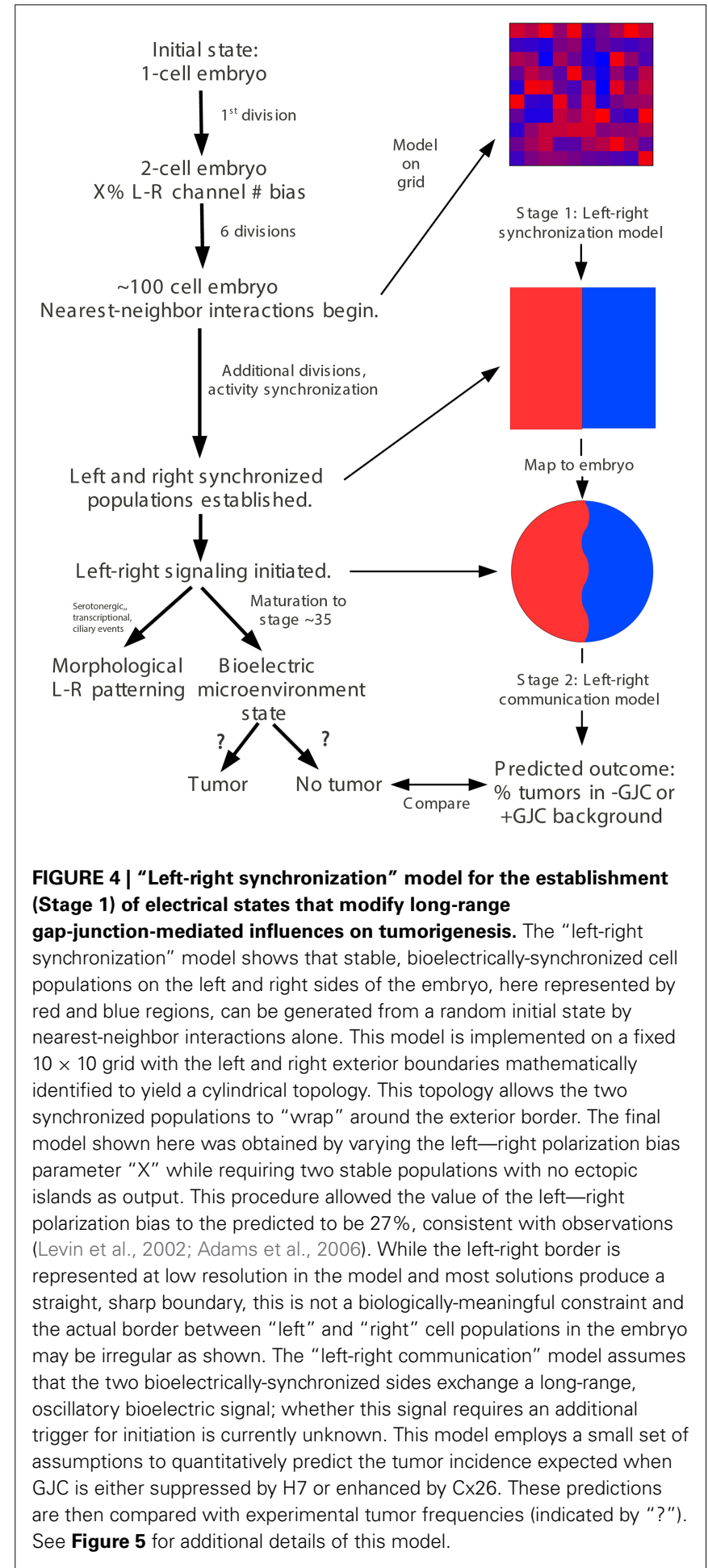

the embryonic animal cap epithelium. Each cell is assumed to communicate only with its four nearest neighbors. Each square of the $10 \times 10$ array (Figure 4) represents one cell at the 100-cell stage; as embryonic cells continue to divide, each square represents the daughter cells in the epithelial "patch" occupied by their progenitor at the 100-cell stage. The left and right boundaries of the planar array are mathematically identified (joined) so that 


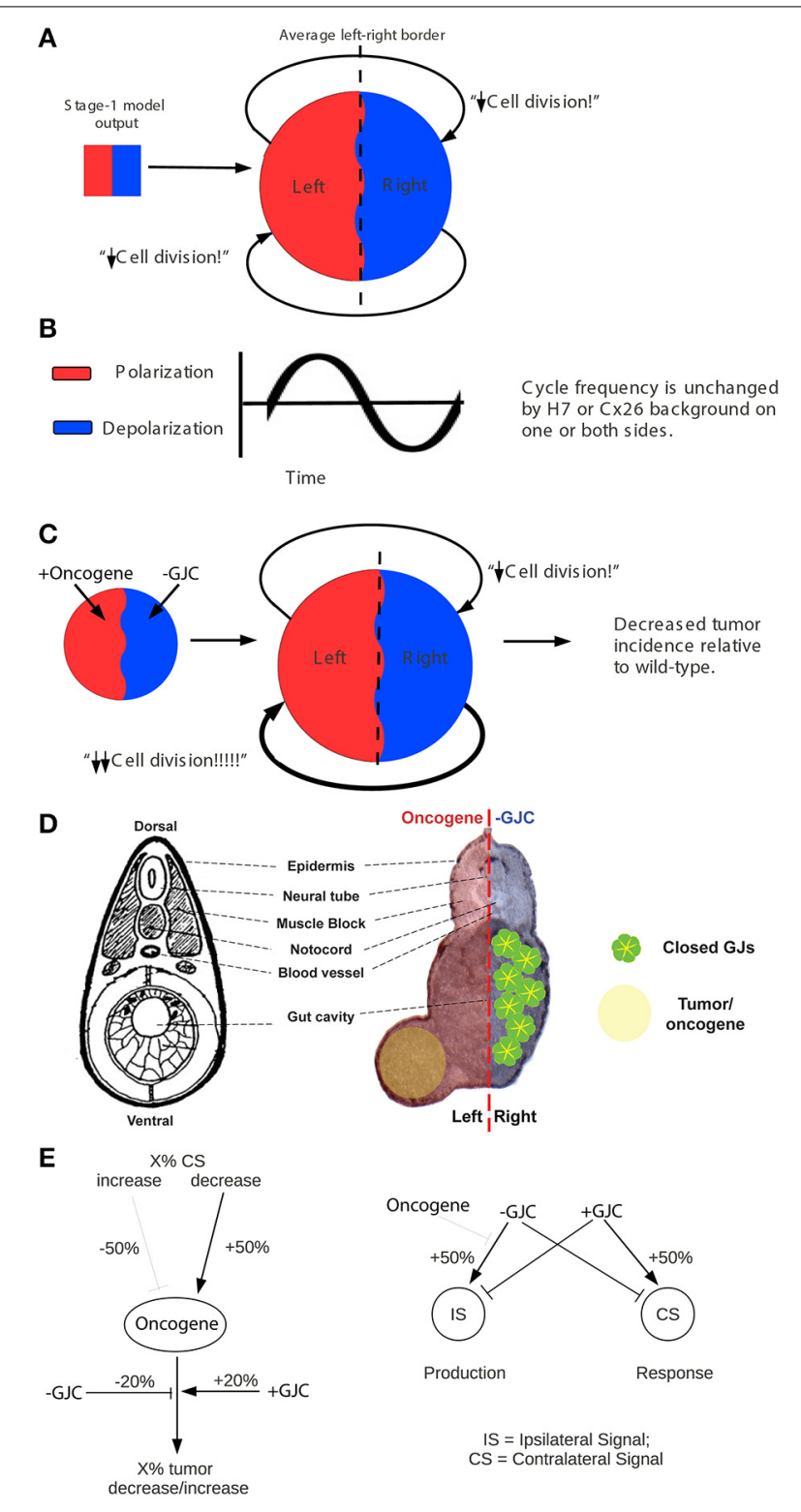

FIGURE 5 | The stage-2 "left-right communication" model for the GJC-mediated effects of $\mathbf{V}_{\text {mem }}$ change on incidence of tumorigenesis. The "left-right communication" model assumes that the left and right sides of the embryo alternately signal each other to turn the rate of cell division down (A) using an oscillation between polarization and depolarization as the long-range bioelectric signal (B). For example, KRAS ${ }^{G 12 D}$ and $\mathrm{H7}$ expressions on opposite sides of the embryo (as shown in Figure 2 treatment $\mathrm{C}$, and sections through a trunk tumor in Panel D) result in an increase in signaling from the $\mathrm{H} 7$-injected side and a consequent suppression in $K R A S^{G 12 D}$-induced tumors on the opposite side $(\mathbf{C}, \mathbf{D})$. The stage-2 model can be visualized as a control network that regulates cellular response to $K R A S^{G 12 D}$ and both the production of ipsilateral signal (IS) and response to contralateral signal (CS). Here unlabeled flat-end arrows indicate a $100 \%$ suppressing effect (E). In this model, gap junctions are a central component, contributing to the long-range dynamics by allowing cells to sense neighbors' $V_{\text {mem }}$. Cellular mechanisms corresponding to these arrows are not yet fully characterized; however, the butyrate-histone deacetylase pathway previously characterized in these embryos (Chernet and Levin, 2013a,b, 2014); is a plausible candidate for transducing the global bioelectrical signal into a local cellular response to $K R A S^{G 12 D}$ transformation. neighborhoods "wrap" around this boundary; the $10 \times 10$ planar array thus has the topology of a cylinder. Making the upper and lower boundaries join also, to form a spherical topology, was found to have no significant effect on the model's behavior.

The initial random state of the $10 \times 10$ array was generated by assigning each square of the array two independent integer values: a random "polarization" value between 0 and 255 and a random "depolarization" value between 0 and 255. These random values were then biased by adding a single bias value both to the random "polarization" values of the squares on the left side of the grid and to the random "depolarization" values of the squares on the right side of the grid. At the 100-cell stage, these distinct values represent the potentially-distinct polarization values of distinct cell-membrane patches within a single cell (Wallace, 2007; Adams and Levin, 2013); their sum represents the average $V_{\text {mem }}$ of that cell. Following the 100-cell stage, these distinct values also represent the potentially-distinct polarization values of distinct daughter cells within an epithelial patch; their sum represents the average $\mathrm{V}_{\text {mem }}$ of that patch. The JavaScript Math.random function was used to generate random values. Cell behavior was modeled by a single local rule uniformly implemented by all squares of the array. On each cycle, each square computed the ratio between the total polarization and depolarization values of its four neighbors. If the ratio was greater than or equal to 1 , the square multiplied its own polarization value by 1.2 and its own depolarization value by 0.8 ; if the ratio was less than 1 , it did the opposite. These multipliers represent the local response of cells to the average bioelectric state of neighboring cells; cells surrounded by polarized cells become more polarized, while cells surrounded by depolarized cells become less polarized. The values of 1.2 and 0.8 were chosen to produce convergence to a stable state within less than 50 cycles on most model runs; small variations in the values of these multipliers had no significant effect on the qualitative behavior of the model.

This first stage of the model represents the dynamics of bioelectric state change in the early embryo. Its prediction that nearest-neighbor interactions are sufficient to drive convergence to a stable bioelectric state is borne out by timelapse videos using voltage-sensitive fluorescent dyes (Adams and Levin, 2012) in Xenopus embryos, which reveal precisely this kind of fluctuation that then settles on a consistent difference in resting potential. Interestingly, the model shows that despite considerable stochastic variability in individual cells' states, such a system consistently converges to a stable state in which the embryo exhibits large regions of identical voltage. In this way, this model for the first time provides an explanation for the observed robustness of bioelectric patterns in development despite the known physiological noise (variability) in individual cells' states.

The model was run until all squares had converged to either fully polarized, defined as a "polarization" value of at least 230 and a "depolarization" value of at most 15 , or fully depolarized, defined with the reverse criteria. Replicate model runs that differed only in their initial random states were evaluated on the basis of two criteria: whether the fraction of either polarized or depolarized cells was greater than $65 \%$, and whether more than one region, accounting for "wraps" around the mathematicallyidentified left and right boundaries, in each state existed, i.e., whether there were "ectopic islands" of polarized or depolarized 
cells. Due to the model's cylindrical topology, regions of one color, representing one polarization, sometimes "wrap" around the mathematically-identified left and right boundaries, i.e., blue squares appear on the left border and/or red squares appear on the right border. A region was considered "ectopic" only if it comprised one or more squares completely surrounded by squares of the opposite color, or surrounded by squares of the opposite color on three sides if the remaining side formed part of the top or bottom border. The requirement that the number of solutions failing to satisfy either of these criteria is negligible $(<3 \%)$ in 100 consecutive runs yields a value of $27 \%$ for the initial, 1 st-division bias in $\mathrm{V}_{\text {mem }}$ among the left and right side blastomeres. This prediction of the model is in accordance with data, since direct measurements of voltage states in early embryos, as well as protein and mRNA analyses show consistent and significant biases in the bioelectric states of 2- or 4-cell frog embryos. Decreasing the percent adjustment in polarization and depolarization values on each cycle downward from $20 \%$ increases the convergence time for this model without qualitatively changing the results, as does running the model on a $20 \times 20$ grid (i.e., 400 squares total).

\section{THE TWO-STAGE QUANTITATIVE MODEL ACCURATELY PREDICTS THE DYNAMIC BETWEEN $\mathbf{V}_{\text {mem, }}$ GJC, AND TUMOR FORMATION}

The existence of bioelectrically-synchronized cell populations on the left and right sides of the embryo that is predicted by the stage-1 model is used by the stage-2, "left-right communication" model to explain the observed results of combining KRAS G12D with either $\mathrm{H} 7$ or $\mathrm{Cx} 26$ (Figure 2). Experimental data show that $35 \%$ of $K R A S^{G 12 D}$-injected embryos produced tumors; compare this to $44 \%$ tumor incidence in embryos injected with $K R A S^{G 12 D}$ on both sides (Figure 2). The non-linearity of tumor incidence by itself is an indication of growth control as a result of cross-talk (i.e., a contralateral signal) between sides A and B. Our stage-2 model is based on two primary assumptions. First, we propose that the $V_{\text {mem }}$ values of the cells on the left and right sides of the embryo oscillate, in synchrony, with cells on the left polarizing when cells on the right depolarize and vice-versa as discussed above. Normal, non-tumor cells respond to the long-range, synchronized, oscillatory bioelectric signal produced by the contralateral side of the embryo (i.e., to the contralateral signal), but not to the long-range signal that they themselves produce (i.e., the ipsilateral signal). We conceptualize this contralateral signal as a cell-division suppressor; the $\mathrm{A}$ and $\mathrm{B}$ sides of the embryo are, on this model, alternately signaling to the other side of the embryo to turn the rate of cell division down (Figure 5A), thus maintaining approximate equality of cell-division rates on the two sides. Increased (decreased) contralateral signal is assumed to decrease (increase) tumor formation probability on a $\%$ for $\%$ basis. Second, we propose that disrupting wild-type GJ with either $\mathrm{H} 7$ or $\mathrm{Cx} 26$ alters both production of and response to the long-range signal. In particular, we assume that the expression of a dominant negative $\mathrm{H} 7$ disrupts response to the contralateral signal (Figures 5C,D) on that side in two ways, by blocking the $\%$ for \% tumor-suppressing capability of increased contralateral signal, and by causing cells in that same region to increase production of their own (ipsilateral) signal by $50 \%$. We assume the presence of tumor cells on either side disables the increased signal production on that side. The model also requires that tumor cells, but not normal cells, on the contralateral side respond to this increased signal by further suppressing cell division; otherwise the division of normal cells would be over-suppressed. This restriction corresponds biologically to a response saturation on the part of normal cells that is disabled in tumor cells. We model Cx26 as having the opposite effect of increasing response to contralateral signal and decreasing production of ipsilateral signal. We also assume that local disruption of GJC by $\mathrm{H} 7$ decreases KRAS ${ }^{G 12 D}$ tumor incidence by $20 \%$, while local enhancement of GJC by Cx26 increases tumor incidence by $20 \%$; these local effects are insensitive to contralateral signal.

With these assumptions, it is straightforward to calculate the results expected from the experimental manipulations shown in Figures 2, 3. A single injection of $K R A S^{G 12 D}$ on one side of the embryo produces 35\% tumors (Figures 2, 3, treatment mode A); this baseline number provides the starting point for calculations. An ipsilateral $\mathrm{H} 7$ background decreases cellular response to $K R A S^{G 12 D}$ by $20 \%$; hence the expected tumor incidence following Figure 2 , treatment mode B is $80 \%$ of $35 \%$, i.e., $28 \%$, a prediction error of only $0.3 \%$ compared to the observed value (Figure 6A, treatment mode B). The more interesting case is a contralateral $\mathrm{H} 7$ background (treatment mode C). Here contralateral H7 causes the contralateral cells to increase signal production by $50 \%$; intuitively, the contralateral cells cannot perceive the signal produced by their neighbors due to disruption of GJC by $\mathrm{H} 7$, so they turn up their own signal production to compensate. The KRAS $S^{G 12 D}$-ipsilateral side, therefore, receives 50\% more contralateral signal. Increased contralateral signal turns KRAS ${ }^{G 12 D}$ response down on a \% for \% basis, so the expected tumor incidence following Figure 2, treatment mode $\mathrm{C}$ is $50 \%$ of $35 \%$, i.e., $17.5 \%$, a prediction error of $2.2 \%$ compared to the observed value. In treatment mode $\mathrm{D}$, both sides of the embryo have an $\mathrm{H} 7$ background. Signal production is increased on the side contralateral to $K R A S^{G 12 D}$, but the tumor-suppressing capability of this increased contralateral signal is disrupted by the ipsilateral H7. The situation is, therefore, the same as in treatment mode A; $K R A S^{G 12 D}$ activity is only decreased by $20 \%$ due to the ipsilateral $\mathrm{H} 7$ background. This prediction of $28 \%$ tumors has a prediction error of only $1.2 \%$ (Figure $6 \mathrm{~A}$, treatment mode D). Treatment modes $\mathrm{E}$ and $\mathrm{F}$ both involve KRAS ${ }^{G 12 D}$ injections on both sides of the embryo; here the $44.9 \%$ tumor incidence observed with treatment mode $\mathrm{G}$ is used as the baseline, i.e., a baseline of $22.45 \%$ tumors is assumed on each of the two sides. With KRAS ${ }^{G 12 D}$ present on both sides, neither side can increase signal production in response to $\mathrm{H} 7$. In treatment mode $\mathrm{E}, \mathrm{H} 7$ is present on one side, so the predicted tumor incidence is $20 \%$ less on that side only; hence the total predicted incidence is $(80 \times 22.45 \%)+$ $22.45 \%=40.4 \%$, a prediction error of $1.7 \%$. In treatment mode F, H7 provides $20 \%$ tumor suppression on both sides, so the total predicted incidence is $(80 \times 22.45 \%) \times 2=35.9 \%$, a prediction error of $2.2 \%$ compared to observations (Figure 6A, treatment mode F). The predictions for Cx26 backgrounds are performed similarly (Figure 6B).

The model assumptions used here effectively specify a control structure (Figure 5E). Each arrow in this structure represents a cellular process regulating response to $K R A S^{G 12 D}, \mathrm{H} 7$, or Cx26. To 


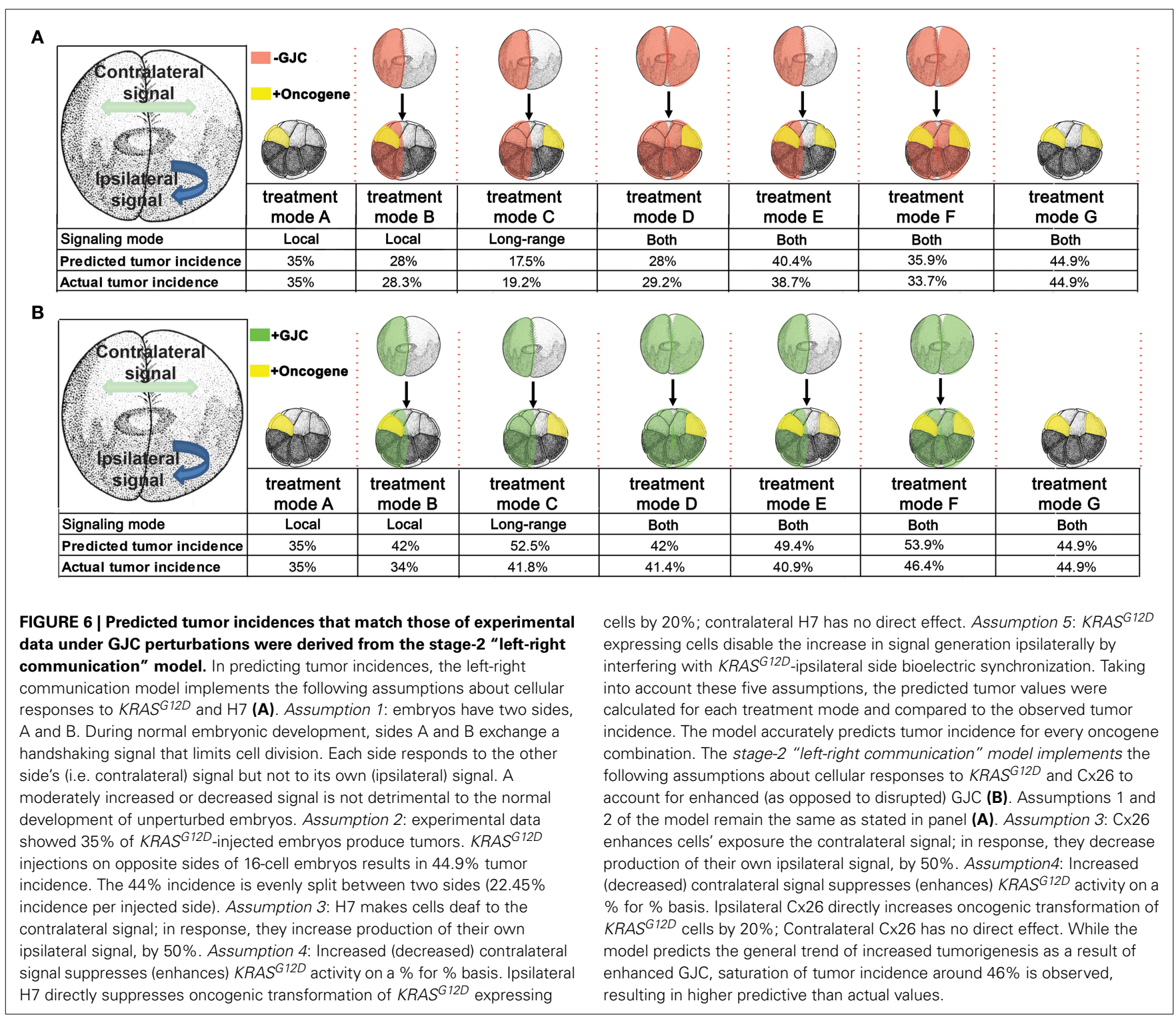

objectively test the apparent ability of the model to correctly predict the observed data (Figures 2, 3), we performed a chi-squared test between the model's predicted outcomes ("expected") and the actual data ("observed") for the various experimental setups. For the H7 injections, $X^{2}=0.547(d f=4), p=0.97$, while for the Cx26 injections, $X^{2}=5.264(d f=4), p=0.26$, thus showing no significant difference between the model's predictions and the experimental data. We conclude that the emergent dynamics of this quantitative model accurately reproduce the complex experimental dataset linking GJC, $\mathrm{V}_{\text {mem }}$ control, and resulting tumor incidence in various spatial configurations.

\section{EXPERIMENTAL CONFIRMATION OF A NOVEL PREDICTION OF THE MODEL: DIFFERENT OUTCOMES OF REAGENT PLACEMENT ALONG THE LEFT-RIGHT vs. DORSO-VENTRAL AXES}

We next tested a novel, surprising prediction of this model that would not have been made without it (by inspection of the experimental data alone): that effectiveness of ion channel injections on tumor suppression will be different depending on which embryonic axis is used to separate the sites of injection.

If two neighboring populations of cells are alternating between polarization and depolarization with opposite phase, so that A-side cells are polarized when B-side cells are depolarized and vice-versa, then the cells forming the border between the two populations must maintain a polarization value very near the average for the two populations. These cells, which form the borders between red and blue in Figure 4 can, therefore, be expected to have qualitatively different behavior from cells away from these borders. Therefore, the model also predicts that the ability of GJC reagents to alter the incidence of tumorigenesis should be different depending on whether the opposite sides of the oncogene/H7 axis are oriented with respect to the left-right or dorso-ventral embryonic axes, since the left-right (LR) axis is the one along which gradients of resting potential are known to exist (Levin et al., 2002; Aw et al., 2008). We tested this prediction (Figure 7), and found that indeed the GJC state is most relevant 


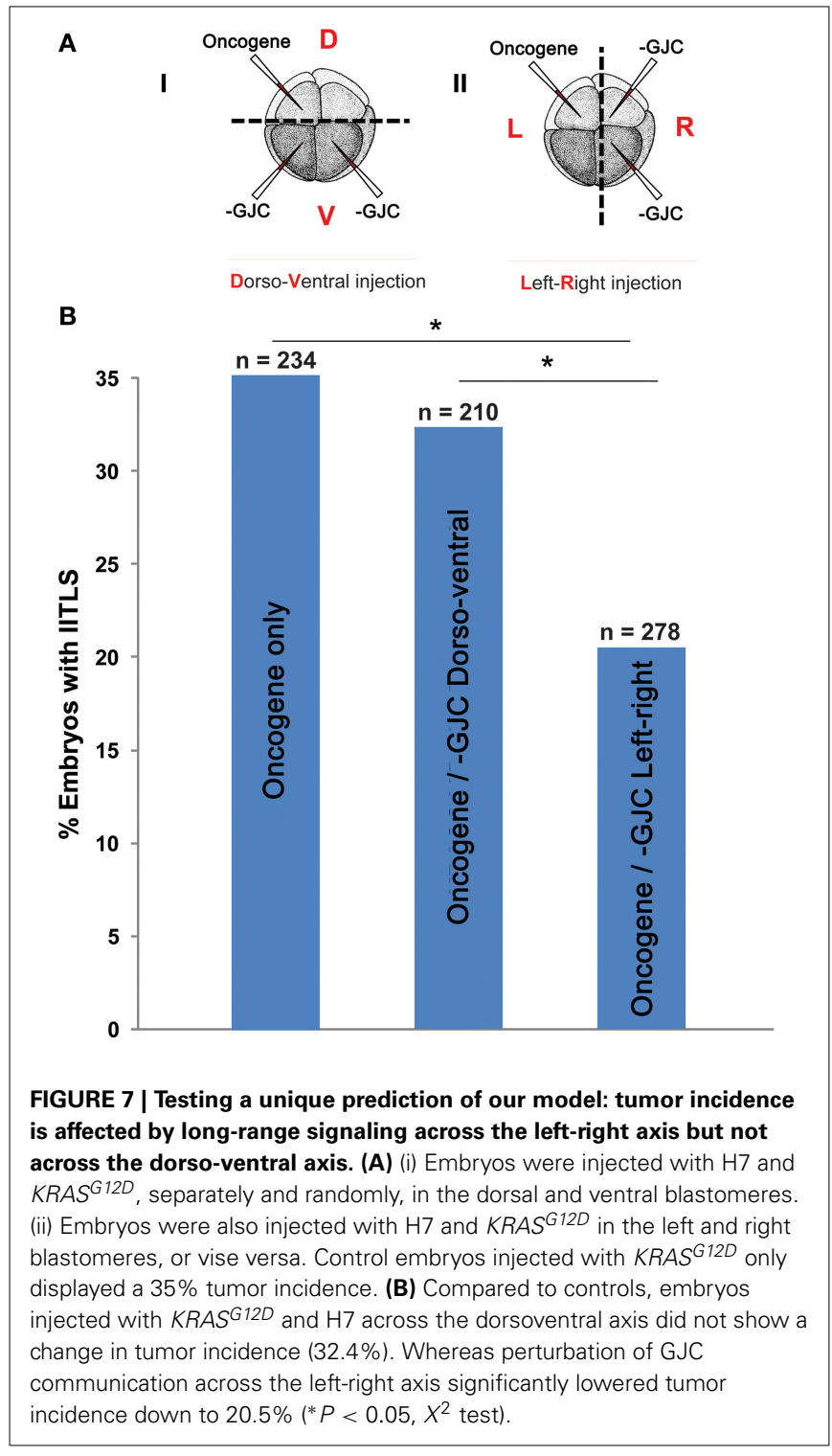

when the opposite-sided distribution of H7-oncogene mRNA coincides with the early embryo's left-right axis. This stage-1 model thus makes unexpected predictions that are supported by experimental test.

\section{DISCUSSION \\ GJ CONNECTIVITY AS A MODULATOR OF VOLTAGE-DEPENDENCE OF TUMOR INDUCTION}

We examined the role of GJC in oncogene-mediated tumorigenesis in Xenopus embryos by targeted molecular-genetic modulation. Injection of mRNA constructs that modify gap junctional states in Xenopus significantly affected the incidence of tumorigenesis, as detected by increases or decreases in tumor formation after oncogene expression (although the absolute magnitude of the effect of GJ-targeting reagents was limited by the need to use low doses of mRNA to avoid disrupting normal development). Reasonably, the reagent that disrupts GJC and the one that enhances GJC affect tumor incidence in opposite directions. However, we were surprised to see that abrogation of GJC actually suppressed the effects of oncogenes, in contrast to prior suggestions that loss of GJC was a hallmark of incipient cancer (Vine and Bertram, 2002; Sirnes et al., 2012). Interestingly, in addition to confirming in vivo the cell-autonomous role inferred for GJCs by cell culture and clinical test data, we found that the greatest impact on tumor incidence occurs when we alter the GJC of cells far away from the tumor. While the microenvironment is increasingly seen to play an important role in cancer (Kenny and Bissell, 2003; Maffini et al., 2005; Hendrix et al., 2007; Kenny et al., 2007; Kasemeier-Kulesa et al., 2008; Tarin, 2012), long-range developmental signals are not yet widely addressed. It should be noted however that two very early workers in the bioelectricity of cancer had previously suggested that such a long-range effect should exist (Burr et al., 1938, 1940; Burr, 1941; Nordenström, 1983).

Targeted expression of wild type connexin Cx26 and dominant negative connexin $\mathrm{H} 7$ allowed us to probe how cell:cell communication affects KRAS ${ }^{G 12 D}$ activity. Induction of GJC host-wide or non-local to $K R A S^{G 12 D}$-expressing cells led to increases in tumor incidence. Enhanced GJC within KRAS ${ }^{G 12 D}$-expressing cells did not affect tumor incidence. While the increases in tumor incidence seen as a result of GJC induction by $\mathrm{Cx} 26$ are significant, the impact of GJC on tumorigenesis was less than expected, partly due to the non-linearity in dose dependence of KRAS ${ }^{G 12 D}$ tumor formation. We demonstrated this non-linearity in dose dependence by showing that doubling KRAS $S^{G 12 D}$ only increases tumor incidence by $9 \%$. Conversely, inhibition of GJC by the dominant negative $\mathrm{H} 7$ led to tumor suppression regardless of where $\mathrm{H} 7$ was introduced. Both local and host-wide GJC significantly reduced tumor incidence, and long-range disruption of GJC had the most impact on tumor incidence, resulting in $15.8 \%$ less embryos with tumor. These results suggest that oncogene-expressing cells utilize the network of GJs between tumors and healthy tissue (Figure 1) to drive tumorigenesis. Most importantly, these data suggest treatment strategies in which oncogene-expressing cells can be junctionally isolated in order to counteract neoplastic transformation.

In contrast to these results, there are significant clinical data implicating GJC as a carcinogenesis suppressor because of its ability to mediate growth control, and that disturbance of GJC between cells is a characteristic of several cancers (Yamasaki, 1990; Rose et al., 1993; Grossman et al., 1994; Hirschi et al., 1996; Yamasaki et al., 1999). However, some tumor cells have been shown to increase expression of functional connexin upon leaving their primary growth sites (Kamibayashi et al., 1995; Zhang et al., 1999). For example, melanoma cells have low levels of Cx26 expression when residing in the epidermal basal layer. However, upon acquiring sustained growth capability, they detach from the epidermis and up-regulate Cx26 expression, which is thought to help them couple with endothelial cells and infiltrate secondary sites (Ito et al., 2000). Overall, the studies presented here highlight scenarios in which enhanced GJC is favorable to tumors and detrimental to the host.

\section{A QUANTITATIVE MODEL INTEGRATING GJ, VOLTAGE, AND ANATOMICAL AXES IN TUMORIGENESIS}

The results presented here revealed that blocking GJC suppressed tumor formation, while promoting GJC enhanced tumor formation. Even more remarkable was the spatial range over 
which tumorigenesis could be affected: modulation of GJC at the maximal possible distance from oncogene expressing cells had the most impact on tumor incidence, suggesting a longrange morphogenetic signal that controls tumor growth. In order to formulate a model that quantitatively predicts and explains this puzzling dataset, we undertook a mechanistic/mathematical approach featuring a reasonable set of assumptions.

We propose that $\mathrm{H} 7$ disrupts the coordination of polarizationdepolarization cycles on the affected side and makes the voltage swings on the affected side bigger; a bigger detected swing suppresses tumor formation on the contralateral side, possibly by affecting the activity of $\mathrm{V}_{\text {mem }}$ dependent channels. Enhancing GJC with Cx26, in contrast, may disrupt the stability of the boundary separating polarized and depolarized domains. In other words, altering local cell-cell synchrony with GJC-altering reagents may prevent cells from responding in a coordinated way to changes in the polarization states of both their near and distant neighbors, and hence disrupt the oscillating "breathing" pattern required for normal control of cell proliferation. This can be attributed to the disruption of multicellular physiological networks established by electrical coupling of gap-junctionally connected cells. One of the interesting aspects of this kind of dynamics is that it is eminently suitable for integration of complex decision-making as would be needed for developmental patterning; for example, the ability of plasmodium organisms to optimize food gathering strategies is currently thought to be implemented by information processing that arises from the integration of oscillatory patterns (Tsuda et al., 2009).

While we do not at this time know the molecular nature of the two signals that implement these dynamics, butyrate and serotonin are good candidates, given previous data on the role of these small signaling molecules in long-range bioelectrical events that regulate cancer (Blackiston et al., 2011; Lobikin et al., 2012; Chernet and Levin, 2013b, 2014). We propose that gap junctions function in this process by regulating ion flow that synchronizes distant cells, enabling global oscillation-the periodic change in the overall electric field within the embryo that provides long-range information exchange. Our model is able to quantitatively account for this surprising dataset and to make new predictions that were verified by experiment. Subsequent work will experimentally test additional predictions of the model. In addition to its implications for control of tumorigenesis, the first phase of the model (establishment of consistent LR gradient from stochastic and highly variable initial $\mathrm{V}_{\text {mem }}$ cell states) has significant implications for the problem of left-right patterning. If correct, it suggests that the focus of new experiments and theory should be not on stable voltage differences arising directly and immediately from early ion channel localization at 4-cell stage, but on understanding the output of a biased dynamical system operating at 128-cell to blastula stages. It is also significant that our model makes experimentally-verified predictions which functionally link left-right asymmetry and cancer. While connections between these two disparate-seeming fields have been suggested previously (McManus, 1992; Sandson et al., 1992; Wan et al., 2011; Wilting and Hagedorn, 2011; Sauer and Klar, 2012; Veltmaat et al., 2013), this is the first quantitative model of the

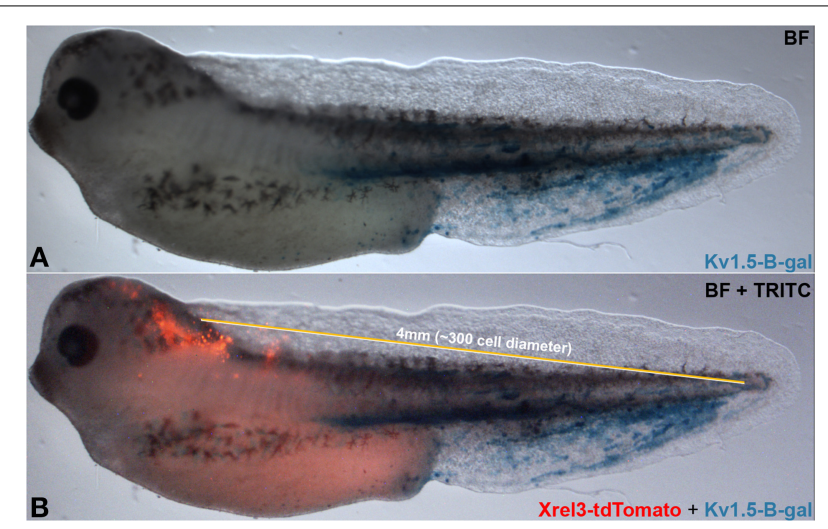

FIGURE 8 | Long-range signaling in the frog embryo.

Oncogene-expressing cells (red, Xrel3-tdTomato) are prevented from forming tumors by hyperpolarizing channel-expressing cells (Kv $1.5-\beta$-gal) located at a distance. Compare (A) without to (B) with Xrel3-tdTomato. The $\sim 4 \mathrm{~mm}$ distance from the center of the tdTomato signal to the farthest point on the tail expressing $\beta$-gal was estimated to be $\sim 300$ cell diameter (average single cell diameter of $13.3 \mu \mathrm{m}$ ).

molecular mechanisms by which these different aspects of pattern regulation are unified.

\section{CONCLUSION AND PERSPECTIVE}

Taken together, our data suggest a complex cross-talk between distant regions of the body that impinge on the stochastic nature of tumorigenesis and physiological dynamics. Additional parameters related to gap junction density include properties such as tissue stiffness, which is known to influence cancer cell behavior and alter cell responses to genetic and epigenetic signals (Bizzarri and Cucina, 2014; Pisanu et al., 2014). Future work will integrate these factors into ever more comprehensive models of tumor formation. While the frog embryo is not considered large by the standards of human medicine (long-range signaling occurs on a scale of from 1.3 to at least $4 \mathrm{~mm}$ ), it represents about 300 cell diameters (a unit of cell diameter $=13.3 \mu \mathrm{m})$ distinctly non-cell-autonomous (Figure 8). Subsequent work will mechanistically test other predictions of our model, by tracking real-time bioelectrical communication through GJs during tumor induction (a technically very challenging task at the edge of current capability). Our data suggest specific tests of this type of signaling in a mammalian model, and imply that biomedical cancer strategies must consider not only the events of the tumor itself and its microenvironment, but perhaps also signals moving to and from quite remote tissues in the body.

\section{ACKNOWLEDGMENTS}

We thank Leonard Zon for the KRAS mutant construct, and David Paul for the H7 and Cx26 constructs. We thank Amber Currier, Erin Switzer, and Amanda Allen for general lab assistance and frog husbandry, and Joan Lemire and Jean-Francois Pare for molecular biology assistance. We thank Stephanie Irvine for helpful suggestions on the computational modeling, Daniel Lobo, Vaibhav Pai, and Gary McDowell for comments on a draft of the manuscript, as well as Douglas Brash and the members of 
the Levin lab for many useful discussions on these topics. Michael Levin gratefully acknowledges support of the G. Harold and Leila Y. Mathers Charitable Foundation.

\section{REFERENCES}

Adams, D. S., and Levin, M. (2012). Measuring resting membrane potential using the fluorescent voltage reporters DiBAC4(3) and CC2-DMPE. Cold Spring Harb. Protoc. 2012, 459-464. doi: 10.1101/pdb.prot067702

Adams, D. S., and Levin, M. (2013). Endogenous voltage gradients as mediators of cell-cell communication: strategies for investigating bioelectrical signals during pattern formation. Cell Tissue Res. 352, 95-122. doi: 10.1007/s00441-0121329-4

Adams, D. S., Robinson, K. R., Fukumoto, T., Yuan, S., Albertson, R. C., Yelick, P., et al. (2006). Early, H+-V-ATPase-dependent proton flux is necessary for consistent left-right patterning of non-mammalian vertebrates. Development 133 1657-1671. doi: 10.1242/dev.02341

Arcangeli, A., Bianchi, L., Becchetti, A., Faravelli, L., Coronnello, M., Mini, E., et al (1995). A novel inward-rectifying $\mathrm{K}+$ current with a cell-cycle dependence governs the resting potential of mammalian neuroblastoma cells. J. Physiol. 489, 455-471. doi: 10.1113/jphysiol.1995.sp021065

Arcangeli, A., Crociani, O., Lastraioli, E., Masi, A., Pillozzi, S., and Becchetti, A. (2009). Targeting ion channels in cancer: a novel frontier in antineoplastic therapy. Curr. Med. Chem. 16, 66-93. doi: 10.2174/0929867097870 02835

Arcangeli, A., Pillozzi, S., and Becchetti, A. (2012). Targeting ion channels in leukemias: a new challenge for treatment. Curr. Med. Chem. 19, 683-696. doi 10.2174/092986712798992093

Aw, S., Adams, D. S., Qiu, D., and Levin, M. (2008). H,K-ATPase protein localization and Kir4.1 function reveal concordance of three axes during early determination of left-right asymmetry. Mech. Dev. 125, 353-372. doi: 10.1016/j.mod.2007.10.011

Barrio, L. C., Capel, J., Jarillo, J. A., Castro, C., and Revilla, A. (1997). Species-specific voltage-gating properties of connexin-45 junctions expressed in Xenopus oocytes. Biophys. J. 73, 757-769. doi: 10.1016/S0006-3495(97) 78108-6

Bennett, M. V. (1997). Gap junctions as electrical synapses. J. Neurocytol. 26, 349-366. doi: 10.1023/A:1018560803261

Bissell, M. J., and Hines, W. C. (2011). Why don't we get more cancer? A proposed role of the microenvironment in restraining cancer progression. Nat. Med. 17, 320-329. doi: 10.1038/nm.2328

Bizzarri, M., and Cucina, A. (2014). Tumor and the microenvironment: a chance to reframe the paradigm of carcinogenesis? Biomed Res. Int. 2014:934038. doi: $10.1155 / 2014 / 934038$

Blackiston, D., Adams, D. S., Lemire, J. M., Lobikin, M., and Levin, M. (2011). Transmembrane potential of GlyCl-expressing instructor cells induces a neoplastic-like conversion of melanocytes via a serotonergic pathway. Dis. Model Mech. 4, 67-85. doi: 10.1242/dmm.005561

Blackiston, D. J., McLaughlin, K. A., and Levin, M. (2009). Bioelectric controls of cell proliferation: ion channels, membrane voltage and the cell cycle. Cell Cycle 8, 3519-3528. doi: 10.4161/cc.8.21.9888

Bregestovski, P., Medina, I., and Goyda, E. (1992). Regulation of potassium conductance in the cellular membrane at early embryogenesis. J. Physiol. Paris 86 , 109-115. doi: 10.1016/S0928-4257(05)80014-2

Bruzzone, R., White, T. W., and Goodenough, D. A. (1996a). The cellular Internet: on-line with connexins. Bioessays 18, 709-718.

Bruzzone, R., White, T. W., and Paul, D. L. (1996b). Connections with connexins: the molecular basis of direct intercellular signaling. Eur. J. Biochem. 238, 1-27.

Burr, H. S. (1941). Changes in the field properties of mice with transplanted tumors. Yale J. Biol. Med. 13, 783-788.

Burr, H. S., Smith, G. M., and Strong, L. C. (1940). Electrometric studies of tumors in mice induced by the external application of benzpyrene. Yale J. Biol. Med. 12, 711-717.

Burr, H. S., Strong, L. C., and Smith, G. M. (1938). Bio-Electric Correlates of Methylcolanthrene-Induced Tumors in mice. Yale J. Biol. Med. 10, 539-544.

Cao, F., Eckert, R., Elfgang, C., Nitsche, J. M., Snyder, S. A., H-ulser, D. F., et al. (1998). A quantitative analysis of connexin-specific permeability differences of gap junctions expressed in HeLa transfectants and Xenopus oocytes. J. Cell Sci. 111(Pt 1), 31-43.
Chernet, B., and Levin, M. (2013a). Endogenous voltage potentials and the microenvironment: bioelectric signals that reveal, induce and normalize cancer. J. Clin. Exp. Oncol. (Suppl 1)S1-002. doi: 10.4172/2324-9110. S1-002

Chernet, B. T., and Levin, M. (2013b). Transmembrane voltage potential is an essential cellular parameter for the detection and control of tumor development in a Xenopus model. Dis. Model Mech. 6, 595-607. doi: 10.1242/dmm. 010835

Chernet, B. T., and Levin, M. (2014). Transmembrane voltage potential of somatic cells controls oncogene-mediated tumorigenesis at long-range. Oncotarget 5, 3287-3306.

Clark, W. H. Jr. (1995). The nature of cancer: morphogenesis and progressive (self)-disorganization in neoplastic development and progression. Acta Oncol. 34, 3-21. doi: 10.3109/02841869509093632

Dale, L., and Slack, J. (1987). Fate map for the 32-cell stage of Xenopus laevis. Development 100, 279-296.

Dean, M. (1998). Cancer as a complex developmental disorder-nineteenth Cornelius P. Rhoads Memorial Award Lecture. Cancer Res. 58, 5633-5636.

Diss, J. K., Stewart, D., Pani, F., Foster, C. S., Walker, M. M., Patel, A., et al (2005). A potential novel marker for human prostate cancer: voltage-gated sodium channel expression in vivo. Prostate Cancer Prostatic Dis. 8, 266-273. doi: 10.1038/sj.pcan.4500796

Donahue, H. J., Saunders, M. M., Li, Z., Mastro, A. M., Gay, C. V., and Welch, D. R. (2003). A potential role for gap junctions in breast cancer metastasis to bone. J. Musculoskelet. Neuronal Interact. 3, 156-161.

Duflot-Dancer, A., Mesnil, M., and Yamasaki, H. (1997). Dominant-negative abrogation of connexin-mediated cell growth control by mutant connexin genes. Oncogene 15, 2151-2158. doi: 10.1038/sj.onc.1201393

Elzarrad, M. K., Haroon, A., Willecke, K., Dobrowolski, R., Gillespie, M. N., and AlMehdi, A. B. (2008). Connexin-43 upregulation in micrometastases and tumor vasculature and its role in tumor cell attachment to pulmonary endothelium. BMC Med. 6:20. doi: 10.1186/1741-7015-6-20

Ewart, J. L., Cohen, M. F., Meyer, R. A., Huang, G. Y., Wessels, A., Gourdie, R. G., et al. (1997). Heart and neural tube defects in transgenic mice overexpressing the Cx43 gap junction gene. Development 124, 1281-1292.

Fraser, S. P., Diss, J. K., Chioni, A. M., Mycielska, M. E., Pan, H., Yamaci, R. F., et al (2005). Voltage-gated sodium channel expression and potentiation of human breast cancer metastasis. Clin. Cancer Res. 11, 5381-5389. doi: 10.1158/10780432.CCR-05-0327

Gee, J., Tanaka, M., and Grossman, H. B. (2003). Connexin 26 is abnormally expressed in bladder cancer. J. Urol. 169, 1135-1137. doi: 10.1097/01.ju.0000041954.91331.df

Goldberg, G. S., Valiunas, V., and Brink, P. R. (2004). Selective permeability of gap junction channels. Biochim. Biophys. Acta 1662, 96-101. doi: 10.1016/j.bbamem.2003.11.022

Goodenough, D. A., Goliger, J. A., and Paul, D. L. (1996). Connexins, connexons, and intercellular communication. Annu. Rev. Biochem. 65, 475-502. doi: 10.1146/annurev.bi.65.070196.002355

Grossman, H. B., Liebert, M., Lee, I. W., and Lee, S. W. (1994). Decreased connexin expression and intercellular communication in human bladder cancer cells. Cancer Res. 54, 3062-3065.

Haass, N. K., Ripperger, D., Wladykowski, E., Dawson, P., Gimotty, P. A., Blome, C., et al. (2010). Melanoma progression exhibits a significant impact on connexin expression patterns in the epidermal tumor microenvironment. Histochem. Cell Biol. 133, 113-124. doi: 10.1007/s00418-009-0654-5

Hendrix, M. J., Seftor, E. A., Seftor, R. E., Kasemeier-Kulesa, J., Kulesa, P. M., and Postovit, L. M. (2007). Reprogramming metastatic tumour cells with embryonic microenvironments. Nat. Rev. Cancer 7, 246-255. doi: 10.1038/nr c2 108

Hirschi, K. K., Xu, C. E., Tsukamoto, T., and Sager, R. (1996). Gap junction genes Cx26 and Cx43 individually suppress the cancer phenotype of human mammary carcinoma cells and restore differentiation potential. Cell Growth Differ. 7, 861-870.

House, C. D., Vaske, C. J., Schwartz, A., Obias, V., Frank, B., Luu, T., et al. (2010) Voltage-gated $\mathrm{Na}+$ channel SCN5A is a key regulator of a gene transcriptional network that controls colon cancer invasion. Cancer Res. 70, 6957-6967. doi: 10.1158/0008-5472.CAN-10-1169

Ito, A., Katoh, F., Kataoka, T. R., Okada, M., Tsubota, N., Asada, H., et al. (2000). A role for heterologous gap junctions between melanoma and 
endothelial cells in metastasis. J. Clin. Invest. 105, 1189-1197. doi: 10.1172/ JCI8257

Jongsma, H. J., and Wilders, R. (2000). Gap junctions in cardiovascular disease. Circ. Res. 86, 1193-1197. doi: 10.1161/01.RES.86.12.1193

Kamibayashi, Y., Oyamada, Y., Mori, M., and Oyamada, M. (1995). Aberrant expression of gap junction proteins (connexins) is associated with tumor progression during multistage mouse skin carcinogenesis in vivo. Carcinogenesis 16, 1287-1297. doi: 10.1093/carcin/16.6.1287

Kasemeier-Kulesa, J. C., Teddy, J. M., Postovit, L. M., Seftor, E. A., Seftor, R. E., Hendrix, M. J., et al. (2008). Reprogramming multipotent tumor cells with the embryonic neural crest microenvironment. Dev. Dyn. 237, 2657-2666. doi: 10.1002/dvdy.21613

Kenny, P. A., and Bissell, M. J. (2003). Tumor reversion: correction of malignant behavior by microenvironmental cues. International journal of cancer. J. Int. Cancer 107, 688-695. doi: 10.1002/ijc.11491

Kenny, P. A., Lee, G. Y., and Bissell, M. J. (2007). Targeting the tumor microenvironment. Front. Biosci. 12, 3468-3474. doi: 10.2741/2327

King, T. J., and Bertram, J. S. (2005). Connexins as targets for cancer chemoprevention and chemotherapy. Biochim. Biophys. Acta 1719, 146-160. doi: 10.1016/j.bbamem.2005.08.012

Krysko, D. V., Leybaert, L., Vandenabeele, P., and D’Herde, K. (2005). Gap junctions and the propagation of cell survival and cell death signals. Apoptosis 10, 459-469. doi: 10.1007/s10495-005-1875-2

Le, X., Langenau, D. M., Keefe, M. D., Kutok, J. L., Neuberg, D. S., and Zon, L. I. (2007). Heat shock-inducible Cre/Lox approaches to induce diverse types of tumors and hyperplasia in transgenic zebrafish. Proc. Natl. Acad. Sci. U.S.A. 104, 9410-9415. doi: 10.1073/pnas.0611302104

Lee, J. R., Derosa, A. M., and White, T. W. (2009). Connexin mutations causing skin disease and deafness increase hemichannel activity and cell death when expressed in Xenopus oocytes. J. Invest. Dermatol. 129, 870-878. doi: 10.1038/jid.2008.335

Levin, M. (2002). Isolation and community: a review of the role of gap-junctional communication in embryonic patterning. J. Membr. Biol. 185, 177-192. doi: 10.1007/s00232-001-0129-7

Levin, M. (2007). Gap junctional communication in morphogenesis. Prog. Biophys. Mol. Biol. 94, 186-206. doi: 10.1016/j.pbiomolbio.2007.03.005

Levin, M. (2011). The wisdom of the body: future techniques and approaches to morphogenetic fields in regenerative medicine, developmental biology and cancer. Regen. Med. 6, 667-673. doi: 10.2217/rme. 11.69

Levin, M. (2012a). Molecular bioelectricity in developmental biology: new tools and recent discoveries: control of cell behavior and pattern formation by transmembrane potential gradients. Bioessays 34, 205-217. doi: $10.1002 /$ bies.201100136

Levin, M. (2012b). Morphogenetic fields in embryogenesis, regeneration, and cancer: non-local control of complex patterning. Biosystems 109, 243-261. doi: 10.1016/j.biosystems.2012.04.005

Levin, M. (2013). Reprogramming cells and tissue patterning via bioelectrical pathways: molecular mechanisms and biomedical opportunities. Wiley Interdiscip. Rev. Syst. Biol. Med. 5, 657-676. doi: 10.1002/wsbm.1236

Levin, M. (2014). Endogenous bioelectrical networks store non-genetic patterning information during development and regeneration. J. Physiol. 592, 2295-2305. doi: 10.1113/jphysiol.2014.271940

Levin, M., and Mercola, M. (1998). Gap junctions are involved in the early generation of left-right asymmetry. Dev. Biol. 203, 90-105. doi: 10.1006/dbio.1998.9024

Levin, M., and Mercola, M. (1999). Gap junction-mediated transfer of left-right patterning signals in the early chick blastoderm is upstream of Shh asymmetry in the node. Development 126, 4703-4714.

Levin, M., Thorlin, T., Robinson, K. R., Nogi, T., and Mercola, M. (2002). Asymmetries in $\mathrm{H}+/ \mathrm{K}+$-ATPase and cell membrane potentials comprise a very early step in left-right patterning. Cell 111, 77-89. doi: 10.1016/S00928674(02)00939-X

Lewalle, J. M., Cataldo, D., Bajou, K., Lambert, C. A., and Foidart, J. M. (1998). Endothelial cell intracellular $\mathrm{Ca} 2+$ concentration is increased upon breast tumor cell contact and mediates tumor cell transendothelial migration. Clin. Exp. Metastasis 16, 21-29. doi: 10.1023/A:1006555 800862
Lobikin, M., Chernet, B., Lobo, D., and Levin, M. (2012). Resting potential, oncogene-induced tumorigenesis, and metastasis: the bioelectric basis of cancer in vivo. Phys. Biol. 9:065002. doi: 10.1088/1478-3975/9/6/ 065002

Loewenstein, W. R. (1979). Junctional intercellular communication and the control of growth. Biochim. Biophys. Acta. 560, 1-65.

Loewenstein, W. R. (1981). Junctional intercellular communication: the cell-to-cell membrane channel. Physiol. Rev. 61, 829-913.

Loewenstein, W. R., and Kanno, Y. (1966). Intercellular communication and the control of tissue growth: lack of communication between cancer cells. Nature 209, 1248-1249. doi: 10.1038/2091248a0

Maffini, M. V., Calabro, J. M., Soto, A. M., and Sonnenschein, C. (2005). Stromal regulation of neoplastic development: age-dependent normalization of neoplastic mammary cells by mammary stroma. Am. J. Pathol. 167, 1405-1410. doi: 10.1016/S0002-9440(10)61227-8

Magnon, C., Hall, S. J., Lin, J., Xue, X., Gerber, L., Freedland, S. J., et al. (2013). Autonomic nerve development contributes to prostate cancer progression. Science 341:1236361. doi: 10.1126/science.1236361

Marongiu, F., Doratiotto, S., Sini, M., Serra, M. P., and Laconi, E. (2012). Cancer as a disease of tissue pattern formation. Prog. Histochem. Cytochem. 47, 175-207. doi: 10.1016/j.proghi.2012.08.001

McCaig, C. D., Rajnicek, A. M., Song, B., and Zhao, M. (2005). Controlling cell behavior electrically: current views and future potential. Physiol. Rev. 85, 943-978. doi: 10.1152/physrev.00020.2004

McCaig, C. D., Song, B., and Rajnicek, A. M. (2009). Electrical dimensions in cell science. J. Cell Sci. 122, 4267-4276. doi: 10.1242/jcs.023564

McManus, I. C. (1992). Reversed cerebral asymmetry and breast cancer. Lancet 339, 523-524. doi: 10.1016/0140-6736(92)90577-P

Mesnil, M., Crespin, S., Avanzo, J. L., and Zaidan-Dagli, M. L. (2005). Defective gap junctional intercellular communication in the carcinogenic process. Biochim. Biophys. Acta 1719, 125-145. doi: 10.1016/j.bbamem.2005. 11.004

Mesnil, M., Piccoli, C., and Yamasaki, H. (1997). A tumor suppressor gene, Cx26, also mediates the bystander effect in HeLa cells. Cancer Res. 57, 2929-2932.

Moody, S. A., and Kline, M. J. (1990). Segregation of fate during cleavage of frog (Xenopus laevis) blastomeres. Anat. Embryol. 182, 347-362. doi: 10.1007/BF02433495

Morokuma, J., Blackiston, D., and Levin, M. (2008). KCNQ1 and KCNE1 $\mathrm{K}+$ channel components are involved in early left-right patterning in Xenopus laevis embryos. Cell. Physiol. Biochem. 21, 357-372. doi: 10.1159/ 000129628

Naoi, Y., Miyoshi, Y., Taguchi, T., Kim, S. J., Arai, T., Tamaki, Y., et al. (2007). Connexin26 expression is associated with lymphatic vessel invasion and poor prognosis in human breast cancer. Breast Cancer Res. Treat. 106, 11-17. doi: $10.1007 / \mathrm{s} 10549-006-9465-8$

Nieuwkoop, P. D., and Faber, J. (1967). Normal Table of Xenopus Laevis (Daudin). Amsterdam: North-Holland Publishing Company.

Nordenström, B. (1983). Biologically Closed Electric Circuits: Clinical, Experimental, and Theoretical Evidence for an Additional Circulatory System. Stockholm: Nordic Medical Publications.

Nuccitelli, R. (2003a). Endogenous electric fields in embryos during development, regeneration and wound healing. Radiat. Prot. Dosimetry. 106, 375-383. doi: 10.1093/oxfordjournals.rpd.a006375

Nuccitelli, R. (2003b). A role for endogenous electric fields in wound healing. Curr. Top. Dev. Biol. 58, 1-26. doi: 10.1016/S0070-2153(03)58001-2

Oviedo, N. J., Morokuma, J., Walentek, P., Kema, I. P., Gu, M. B., Ahn, J. M., et al. (2010). Long-range neural and gap junction protein-mediated cues control polarity during planarian regeneration. Dev. Biol. 339, 188-199. doi: 10.1016/j.ydbio.2009.12.012

Palacios-Prado, N., and Bukauskas, F. F. (2009). Heterotypic gap junction channels as voltage-sensitive valves for intercellular signaling. Proc. Natl. Acad. Sci. U.S.A. 106, 14855-14860. doi: 10.1073/pnas 0901923106

Pardo, L. A., and Stuhmer, W. (2014). The roles of $\mathrm{K}(+)$ channels in cancer. Nat. Rev. Cancer 14, 39-48. doi: 10.1038/nrc3635

Paul, D. L., Yu, K., Bruzzone, R., Gimlich, R. L., and Goodenough, D. A. (1995). Expression of a dominant negative inhibitor of intercellular communication 
in the early Xenopus embryo causes delamination and extrusion of cells. Development 121, 371-381.

Pawlowski, A., and Weddell, G. (1967). Induction of tumours in denervated skin. Nature 213, 1234-1236. doi: 10.1038/2131234a0

Pereda, A. E., Curti, S., Hoge, G., Cachope, R., Flores, C. E., and Rash, J. E. (2013). Gap junction-mediated electrical transmission: regulatory mechanisms and plasticity. Biochim. Biophys. Acta 1828, 134-146. doi: 10.1016/j.bbamem. 2012.05.026

Pierce, G. B., and Speers, W. C. (1988). Tumors as caricatures of the process of tissue renewal: prospects for therapy by directing differentiation. Cancer Res. 48, 1996-2004.

Pisanu, M. E., Noto, A., De Vitis, C., Masiello, M. G., Coluccia, P., Proietti, S., et al. (2014). Lung cancer stem cell lose their stemness default state after exposure to microgravity. Biomed Res. Int. 2014:470253. doi: 10.1155/2014/ 470253

Qiu, D., Cheng, S. M., Wozniak, L., McSweeney, M., Perrone, E., and Levin, M. (2005). Localization and loss-of-function implicates ciliary proteins in early, cytoplasmic roles in left-right asymmetry. Dev. Dyn. 234, 176-189. doi: 10.1002/dvdy.20509

Rabionet, R., Gasparini, P., and Estivill, X. (2000). Molecular genetics of hearing impairment due to mutations in gap junction genes encoding beta connexins. Hum. Mut. 16, 190-202. doi: 10.1002/1098-1004(200009)16:3<190::AIDHUMU2 $>3.0 . C O ; 2-\mathrm{I}$

Rash, J. E., Curti, S., Vanderpool, K. G., Kamasawa, N., Nannapaneni, S., Palacios-Prado, N., et al. (2013). Molecular and functional asymmetry at a vertebrate electrical synapse. Neuron 79, 957-969. doi: 10.1016/j.neuron.2013. 06.037

Rose, B., Mehta, P. P., and Loewenstein, W. R. (1993). Gap-junction protein gene suppresses tumorigenicity. Carcinogenesis 14, 1073-1075. doi 10.1093/carcin/14.5.1073

Rose, S. M., and Wallingford, H. M. (1948). Transformation of renal tumors of frogs to normal tissues in regenerating limbs of salamanders. Science 107, 457.

Rubin, H. (2006). What keeps cells in tissues behaving normally in the face of myriad mutations? Bioessays 28, 515-524. doi: 10.1002/bies. 20403

Ruch, R. J., and Trosko, J. E. (2001). Gap-junction communication in chemical carcinogenesis. Drug Metab. Rev. 33, 117-124. doi: 10.1081/DMR-1000 00137

Saito-Katsuragi, M., Asada, H., Niizeki, H., Katoh, F., Masuzawa, M., Tsutsumi, M., et al. (2007). Role for connexin 26 in metastasis of human malignant melanoma: communication between melanoma and endothelial cells via connexin 26 . Cancer 110, 1162-1172. doi: 10.1002/cncr.22894

Sandson, T. A., Wen, P. Y., and LeMay, M. (1992). Reversed cerebral asymmetry in women with breast cancer. Lancet 339, 523-524. doi: 10.1016/01406736(92)90341-Y

Sauer, S., and Klar, A. J. (2012). Left-right symmetry breaking in mice by left-right dynein may occur via a biased chromatid segregation mechanism, without directly involving the Nodal gene. Front. Oncol. 2:166. doi: $10.3389 /$ fonc. 2012.00166

Scharrer, B. (1953). Insect tumors induced by nerve severance: incidence and mortality. Cancer Res. 13, 73-76.

Sirnes, S., Bruun, J., Kolberg, M., Kjenseth, A., Lind, G. E., Svindland, A., et al. (2012). Connexin 43 acts as a colorectal cancer tumor suppressor and predicts disease outcome. International journal of cancer. J. Int. Cancer 131, 570-581. doi: $10.1002 /$ ijc. 26392

Sive, H., Grainger, R. M., and Harland, R. M. (2000). Early Development of Xenopus Laevis: A Laboratory Manual. New York, NY: Cold Spring Harbor Laboratory Press.

Soroceanu, L., Manning, T. J. Jr., and Sontheimer, H. (2001). Reduced expression of connexin-43 and functional gap junction coupling in human gliomas. GLIA 33, 107-117. doi: 10.1002/1098-1136(200102)33:2<107::AIDGLIA1010>3.0.CO;2-4

Stoletov, K., Strnadel, J., Zardouzian, E., Momiyama, M., Park, F. D., Kelber, J. A., et al. (2013). Role of connexins in metastatic breast cancer and melanoma brain colonization. J. Cell Sci. 126, 904-913. doi: 10.1242/jcs.112748

Swenson, K. I., Jordan, J. R., Beyer, E. C., and Paul, D. L. (1989). Formation of gap junctions by expression of connexins in Xenopus oocyte pairs. Cell 57, 145-155. doi: 10.1016/0092-8674(89)90180-3
Talbot, J., Brion, R., Picarda, G., Amiaud, J., Chesneau, J., Bougras, G., et al. (2013). Loss of connexin 43 expression in Ewing's sarcoma cells favors the development of the primary tumor and the associated bone osteolysis. Biochim. Biophys. Acta 1832, 553-564. doi: 10.1016/j.bbadis.2013.01.001

Tarin, D. (2012). Clinical and biological implications of the tumor microenvironment. Cancer Microenviron. 5, 95-112. doi: 10.1007/s12307-0120099-6

Temme, A., Buchmann, A., Gabriel, H. D., Nelles, E., Schwarz, M., and Willecke, K. (1997). High incidence of spontaneous and chemically induced liver tumors in mice deficient for connexin32. Curr. Biol. 7, 713-716. doi: 10.1016/S09609822(06)00302-2

Than, B. L., Goos, J. A., Sarver, A. L., O'Sullivan, M. G., Rod, A., Starr, T. K., et al. (2014). The role of KCNQ1 in mouse and human gastrointestinal cancers. Oncogene 33, 3861-3868. doi: 10.1038/onc.2013.350

Trosko, J. E. (2005). The role of stem cells and gap junctions as targets for cancer chemoprevention and chemotherapy. Biomed. Pharmacother. 59(Suppl. 2), S326-S331. doi: 10.1016/S0753-3322(05)80065-4

Tseng, A., and Levin, M. (2013). Cracking the bioelectric code: probing endogenous ionic controls of pattern formation. Commun. Integr. Biol. 6, 1-8. doi: $10.4161 / \mathrm{cib} .22595$

Tsonis, P. A. (1987). Embryogenesis and carcinogenesis: order and disorder. Anticancer Res. 7, 617-623.

Tsuda, S., Artmann, S., and Zauner, K.-P. (2009). "The phi-bot:a robot controlled by a slime, mould," in Artificial Life Models in Hardware, eds A. Adamatzky and M. Komosinski (London: Springer), 213-232.

Vega, A. V., Avila, G., and Matthews, G. (2013). Interaction between the transcriptional corepressor Sin3B and voltage-gated sodium channels modulates functional channel expression. Sci. Rep. 3:2809. doi: 10.1038/srep 02809

Veltmaat, J. M., Ramsdell, A. F., and Sterneck, E. (2013). Positional variations in mammary gland development and cancer. J. Mammary Gland Biol. Neoplasia 18, 179-188. doi: 10.1007/s10911-013-9287-3

Vine, A. L., and Bertram, J. S. (2002). Cancer chemoprevention by connexins. Cancer Metastasis Rev. 21, 199-216. doi: 10.1023/A:1021250 624933

Wallace, R. (2007). Neural membrane microdomains as computational systems: toward molecular modeling in the study of neural disease. Biosystems 87, 20-30. doi: 10.1016/j.biosystems.2006.02.012

Wan, L. Q., Ronaldson, K., Park, M., Taylor, G., Zhang, Y., Gimble, J. M., et al. (2011). Micropatterned mammalian cells exhibit phenotype-specific left-right asymmetry. Proc. Natl. Acad. Sci. U.S.A. 108, 12295-12300. doi: 10.1073/pnas.1103834108

Warner, A. (1992). Gap junctions in development-a perspective. Semin. Cell Biol. 3, 81-91. doi: 10.1016/S1043-4682(10)80009-1

Wilting, J., and Hagedorn, M. (2011). Left-right asymmetry in embryonic development and breast cancer: common molecular determinants? Curr. Med. Chem. 18, 5519-5527. doi: $10.2174 / 092986711798347252$

Wong, R. C., Pera, M. F., and Pebay, A. (2008). Role of gap junctions in embryonic and somatic stem cells. Stem Cell Rev. 4, 283-292. doi: 10.1007/s12015-0089038-9

Yamasaki, H. (1990). Gap junctional intercellular communication and carcinogenesis. Carcinogenesis 11, 1051-1058. doi: 10.1093/carcin/11.7.1051

Yamasaki, H., Krutovskikh, V., Mesnil, M., Tanaka, T., Zaidan-Dagli, M., and Omori, Y. (1999). Role of connexin (gap junction) genes in cell growth control and carcinogenesis. Comptes Rendus de l Acad. des Sci. 322, 151-159. doi: 10.1016/S0764-4469(99)80038-9

Yamasaki, H., Mesnil, M., Omori, Y., Mironov, N., and Krutovskikh, V. (1995). Intercellular communication and carcinogenesis. Mut. Res. 333, 181-188. doi: 10.1016/0027-5107(95)00144-1

Yang, M., and Brackenbury, W. J. (2013). Membrane potential and cancer progression. Front. Physiol. 4:185. doi: 10.3389/fphys.2013.00185

Yildirim, S., Altun, S., Gumushan, H., Patel, A., and Djamgoz, M. B. (2012). Voltage-gated sodium channel activity promotes prostate cancer metastasis in vivo. Cancer Lett. 323, 58-61. doi: 10.1016/j.canlet. 2012.03.036

Zhang, W., Couldwell, W. T., Simard, M. F., Song, H., Lin, J. H., and Nedergaard, M. (1999). Direct gap junction communication between malignant glioma cells and astrocytes. Cancer Res. 59, 1994-2003. 
Zhang, W., DeMattia, J. A., Song, H., and Couldwell, W. T. (2003). Communication between malignant glioma cells and vascular endothelial cells through gap junctions. J. Neurosurg. 98, 846-853. doi: 10.3171/jns.2003.98. 4.0846

Zhao, M., Chalmers, L., Cao, L., Vieira, A. C., Mannis, M., and Reid, B. (2012). Electrical signaling in control of ocular cell behaviors. Prog. Ret. Eye Res. 31, 65-88. doi: 10.1016/j.preteyeres.2011.10.001

Zoidl, G., and Dermietzel, R. (2010). Gap junctions in inherited human disease. Pflugers Arch. 460, 451-466. doi: 10.1007/s00424-010-0789-1

Conflict of Interest Statement: The authors declare that the research was conducted in the absence of any commercial or financial relationships that could be construed as a potential conflict of interest.
Received: 01 September 2014; accepted: 18 December 2014; published online: 19 January 2015.

Citation: Chernet BT, Fields $C$ and Levin M (2015) Long-range gap junctional signaling controls oncogene-mediated tumorigenesis in Xenopus laevis embryos. Front. Physiol. 5:519. doi: 10.3389/fphys.2014.00519

This article was submitted to Biophysics, a section of the journal Frontiers in Physiology.

Copyright (c) 2015 Chernet, Fields and Levin. This is an open-access article distributed under the terms of the Creative Commons Attribution License (CC BY). The use, distribution or reproduction in other forums is permitted, provided the original author(s) or licensor are credited and that the original publication in this journal is cited, in accordance with accepted academic practice. No use, distribution or reproduction is permitted which does not comply with these terms. 